\title{
Investigation on the Effect of Dynamic Frequency on Fracture Evolution in Preflawed Rock under Multistage Cyclic Loads: Insight from Acoustic Emission Monitoring
}

\author{
H. J. Meng $\mathbb{D}^{1},{ }^{1}$ Y. Wang $\mathbb{D}^{2},{ }^{2}$ B. Zhang, ${ }^{3}$ and S. H. Gao ${ }^{2}$ \\ ${ }^{1}$ Key Laboratory of Neotectonic Movement and Geohazard, Institute of Geomechanics, Chinese Academy of Geological Sciences, \\ Beijing 100081, China \\ ${ }^{2}$ Beijing Key Laboratory of Urban Underground Space Engineering, Department of Civil Engineering, School of Civil \& \\ Resource Engineering, University of Science \& Technology Beijing, Beijing 100083, China \\ ${ }^{3}$ Architectural Engineering College, Guizhou Minzu University, Guiyang 550025, China \\ Correspondence should be addressed to Y. Wang; good541571889@126.com
}

Received 8 September 2020; Revised 28 September 2020; Accepted 7 October 2020; Published 30 October 2020

Academic Editor: Chun Zhu

Copyright (c) 2020 H. J. Meng et al. This is an open access article distributed under the Creative Commons Attribution License, which permits unrestricted use, distribution, and reproduction in any medium, provided the original work is properly cited.

\begin{abstract}
This study is aimed at revealing the effect of dynamic loading frequency on the fracture evolution behavior in preflawed rock samples under multistage cyclic loading conditions. The fracture evolution characteristics were investigated using stress-strain descriptions and in situ acoustic emission techniques. It is shown that rock strength, deformation, AE pattern, and fatigue life are strongly affected by the applied dynamic loading frequency. Rock fatigue strength and lifetime increase with the increase of dynamic loading frequency. The AE count and energy output both increase with the increase of the applied loading frequency. Six kinds of cracking modes were revealed by AE spectral frequency analysis. It is shown that large-scaled cracks are easy to be formed for rock subjected to high-frequency loads, reflected as the deceasing of AE signals with high-frequency-high-amplitude signal feature. It is suggested that applied dynamic loading frequency has obvious impact on the crack coalescence at the rock bridge segment. The testing results are helpful to enhance the cognitive of the influence of dynamic frequency on the crack communication behavior and can be expected to predict the stability of rock mass structures where rock mass is subjected to fatigue loading.
\end{abstract}

\section{Introduction}

Many types of loads applied to rock mass are cyclic essentially, including the human-induced engineering loads and the environmental loads. Cyclic rock mechanical behaviors involve many kinds of rock engineering, such as mining engineering, oil-gas development engineering, underground excavation engineering, pumped storage hydroelectricity engineering, and oil and gas storage engineering [1]. On the stress disturbance on rock damage accumulation, the disturbed loads are usually equivalent to cyclic/fatigue loads. Considerable efforts have been performed to investigate the cyclic loading on rock mechanical properties. Among these studies, the impacts of cyclic loading on rock structure deterioration were widely studied [2-6]. The interactions between the human activities and rock engineering constructions highlight the importance of investigating the cyclic behavior of rock. When rocks experience cyclic and especially fatigue loading, several different kinds of dynamic disaster (e.g., rock blasting, collapse, and landslides) phenomena occur along with the disturbance, which influences the long-time stability of rock structures and rock engineering [2]. Therefore, investigation of the geomechanical properties of rock that are subjected to cyclic and fatigue loading is always important to ensure the long-term reliability of the rock structure in mining and civil engineering.

The rock fatigue characteristics have been revealed in the laboratory by many scholars. As for the fatigue dynamic behaviors of rock mass, the stress amplitude and loading frequency are always the main study inspects [6-11]. Singh 


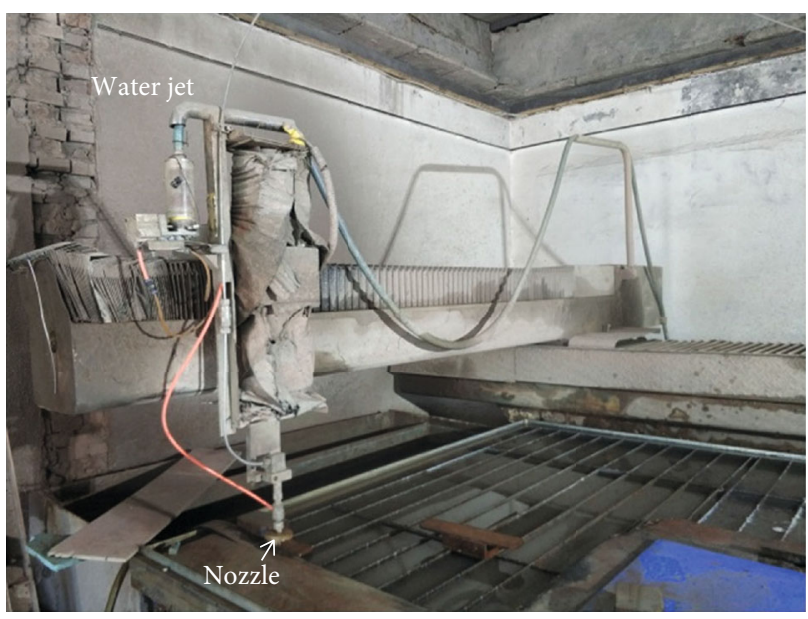

(a)

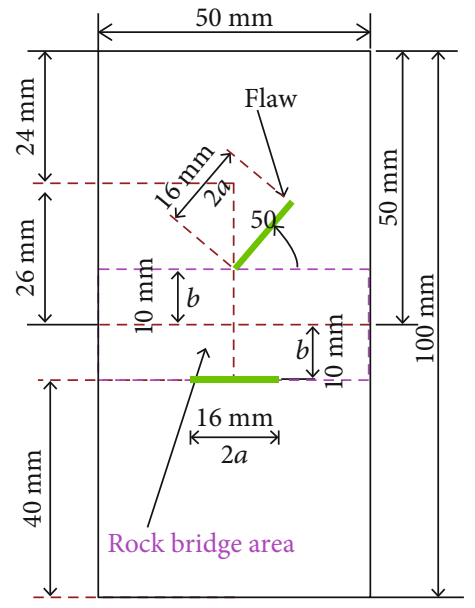

(b)

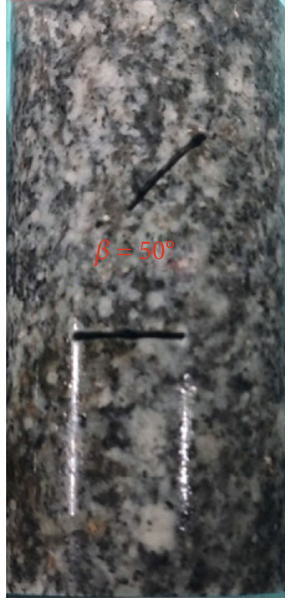

(c)

FIGURE 1: Preparation of the preflawed granite samples. (a) A water jet machine used to cut two flaws in intact rock. (b) Geometry size of the two flaws in rock samples. (c) Typical rock samples with an approach angle of $50^{\circ}$ used to conduct mechanical test.

$[12,13]$ conducted systematic fatigue tests on granite and found that fatigue lifetime decreases with increasing stress amplitude. Ge [14] and Ge et al. [15] found that a fatigue threshold value exists for the rock, and the rock can be damaged and failed when the stress amplitude exceeds the threshold. They also found that the fatigue threshold is close to the crack damage stress on the static stress-strain curve. Bagde and Petroš [9] tested intact sandstone samples from a coal mine under cyclic loading and found that loading frequency and stress amplitude have a great influence on the rock dynamic behavior. They also pointed out that the loading waveforms have a strong influence on the damage accumulation under cyclic loading conditions. Fuenkajorn and Phueakphum [16] conducted a triaxial circulation test and established the relationship between the rock strength and cyclic loading number. In addition, they found that the modulus of elasticity remained almost constant until the specimen failed. Liu and He [10] studied the effect of confining pressure on cyclic mechanical properties of intact rock samples and proposed a residual strain method to describe initial fatigue damage and damage extension. Wang et al. [17] studied the fatigue behavior of granite under triaxial compression and found that the use of axial residual strain can well describe the fatigue behavior of rock. Roberts et al. [18] studied the creep and expansion behavior of salt rock under fatigue load, revealing the deformation characteristics of salt rock. Taheri et al. [19] conducted cyclic loading experiments on lignite and found that the mechanical properties of lignite were not significantly affected by cyclic loading and cumulative irreversible axial strain. Fan et al. [20] conducted discontinuous cyclic loading tests on salt and revealed the differential fatigue life of salt in comparison with continuous cyclic loading tests. Peng et al. [21] investigated the mechanism of loading frequency on sandstone and found that increasing the frequency can improve the strength of rock. Wang et al. [3] revealed the anisotropic fatigue behavior of interbedded marble subjected to constant stress amplitude loading condition; they found that the interbedded structure influences the final fracture morphology of rock. Wang et al. [4] conducted a triaxial fatigue cyclic-confining pressure unloading test on marble; the impact of the rock internal structure on energy evolution and fracture pattern has been investigated.

After the literature review, the influences of dynamic frequency on fatigue mechanical behaviors of rock are limited to constant stress amplitude loading conditions. However, in actual rock engineering, especially in the mining or civil engineering, the rock is subjected to frequent stress disturbance with variable stress amplitudes [17, 18]. For example, in underground mining, the cyclic loading generated by blasting also exerts multilevel effects on a certain rock structure; in open pit mining, with the continuous excavation of the rock slope, the applied stress amplitude is varied and not constant $[22,23]$, that is to say, rock accumulative damage resulting from the increasing amplitude cyclic-stress conditions alters the rock structure, and the final failure of rock body; in a hydropower station, the cyclic loads generated by the rise and fall of the water level are multilevel; in a railway or highway, the cyclic loading generated by the increase and decrease of load capacity and also the number of vehicles is multilevel. As a result, to explore the rock fatigue behaviors under varied stress amplitude within the whole fracturing process is necessary. Moreover, the failure morphology of the rock after fatigue loading experiment can only be observed from the rock surface, the internal crack pattern is difficult to detect, and the mesoscopic failure mechanism is not well understood. Moreover, due to the influence of farfield blast vibration and the vehicle loading, the dynamic frequency acted on the rock is varied and not constant. Therefore, investigating the influence of dynamic frequency on a rock subjected to increasing amplitude cyclic loading is necessary and crucial in rock engineering.

In this work, a series of increasing amplitude cyclic loading experiments with different dynamic frequencies have been performed on granite samples that contain two flaws. The approach angle of the two flaws is designed as $50^{\circ}$ 

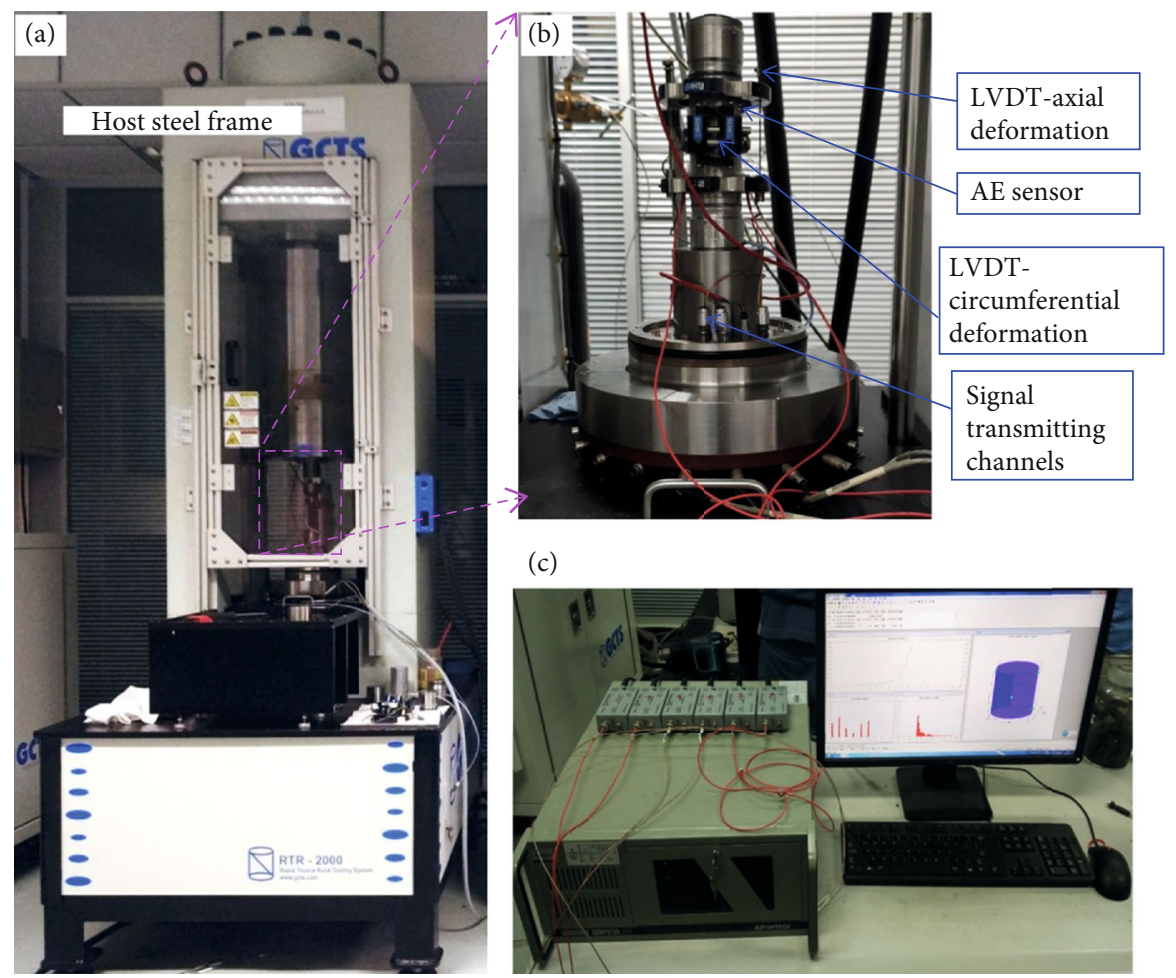

(c)

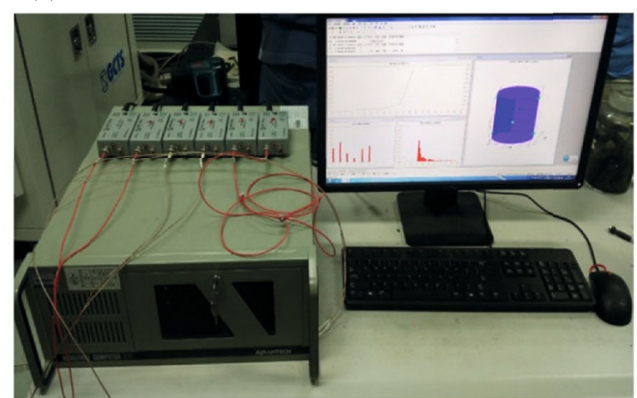

FIGURE 2: Testing system for the preflawed granite samples: (a) GCTS RTR 2000 rock mechanics machine; (b) LVDT system; (c) PAC-AE system.

TABLE 1: Rock loading path description in the cyclic loading tests.

\begin{tabular}{|c|c|c|c|c|c|c|c|c|}
\hline Group & Sample ID & $\begin{array}{c}L \times d \\
(\mathrm{~mm} \times \mathrm{mm})\end{array}$ & $\begin{array}{l}\text { Mass } \\
(\mathrm{g})\end{array}$ & $\begin{array}{l}\text { Peak strength } \\
\qquad(\mathrm{MPa})\end{array}$ & $\begin{array}{l}\text { Loading } \\
\text { cycles }\end{array}$ & $\begin{array}{l}\text { Loading } \\
\text { frequency } \\
(\mathrm{Hz})\end{array}$ & $\begin{array}{l}\text { Lower limit stress } \\
\qquad(\mathrm{MPa})\end{array}$ & Loading and unloading conditions \\
\hline \multirow{2}{*}{ I } & UC-1 & $100.25 \times 49.45$ & 540.6 & 70.26 & - & - & - & \multirow{2}{*}{$\begin{array}{l}\text { Uniaxial, monotonous } \\
\text { compression, } 0.06 \mathrm{~mm} / \mathrm{min}\end{array}$} \\
\hline & UC-2 & $100.03 \times 49.94$ & 540.3 & 73.09 & - & - & - & \\
\hline \multirow{8}{*}{ II } & GIAF-1 & $99.90 \times 49.44$ & 542.8 & 64.185 & 150 & 0.04 & 5 & \multirow{8}{*}{$\begin{array}{l}\text { Uniaxial cyclic, dynamic loading, } \\
\text { increasing amplitude stress of } \\
10 \mathrm{MPa}, 30 \text { cycles were performed } \\
\text { for each fatigue loading stage }\end{array}$} \\
\hline & GIAF-2 & $100.01 \times 49.61$ & 540.6 & 63.125 & 141 & 0.04 & 5 & \\
\hline & GIAF-3 & $100.04 \times 49.38$ & 543.8 & 65.846 & 162 & 0.10 & 5 & \\
\hline & GIAF-4 & $100.05 \times 49.95$ & 543.1 & 64.534 & 160 & 0.10 & 5 & \\
\hline & GIAF-5 & $100.11 \times 50.02$ & 536.4 & 65.761 & 177 & 0.50 & 5 & \\
\hline & GIAF-6 & $100.72 \times 49.82$ & 537.8 & 65.557 & 174 & 0.50 & 5 & \\
\hline & GIAF-7 & $99.90 \times 49.77$ & 533.2 & 73.428 & 190 & 1.00 & 5 & \\
\hline & GIAF-8 & $100.08 \times 49.91$ & 536.8 & 75.126 & 192 & 1.00 & 5 & \\
\hline
\end{tabular}

according to the rock bridge structure in the open pit slope. The fracture evolution of the granite was monitored by acoustic emission (AE), and also, the final crack coalescence pattern was visualized by three-dimensional CT scanning techniques. This work is focused on revealing the effects of the dynamic loading frequency on the fracturing evolution of granite that is subjected to increasing amplitude cyclic loading paths; the results are helpful to understanding the deterioration mechanism of rock mass under stress disturbance conditions.

\section{Materials and Methods}

2.1. Rock Materials and Sample Preparation. The rock material was obtained from an open pit slope of the Xinjiang Hejing Beizhan iron mine, located at the Xinjiang province, northwest of China. From the observation of the rock mass, three sets of joints exist at the open pit slope; a typical locking type structure (i.e., rock bridge structure) can be observed from the outcrop. The coalescence of the locking segment has a strong effect on the stability of the rock slope. After field 

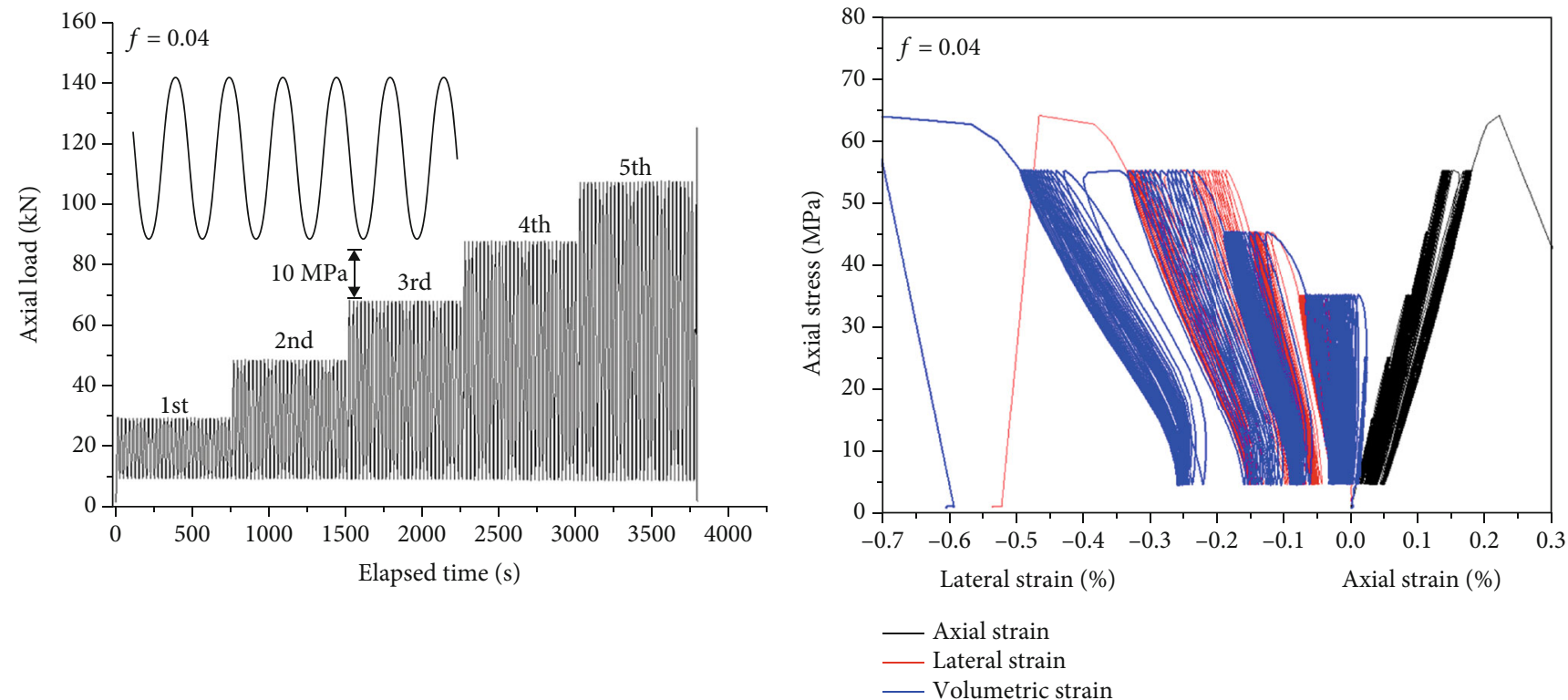

(a)
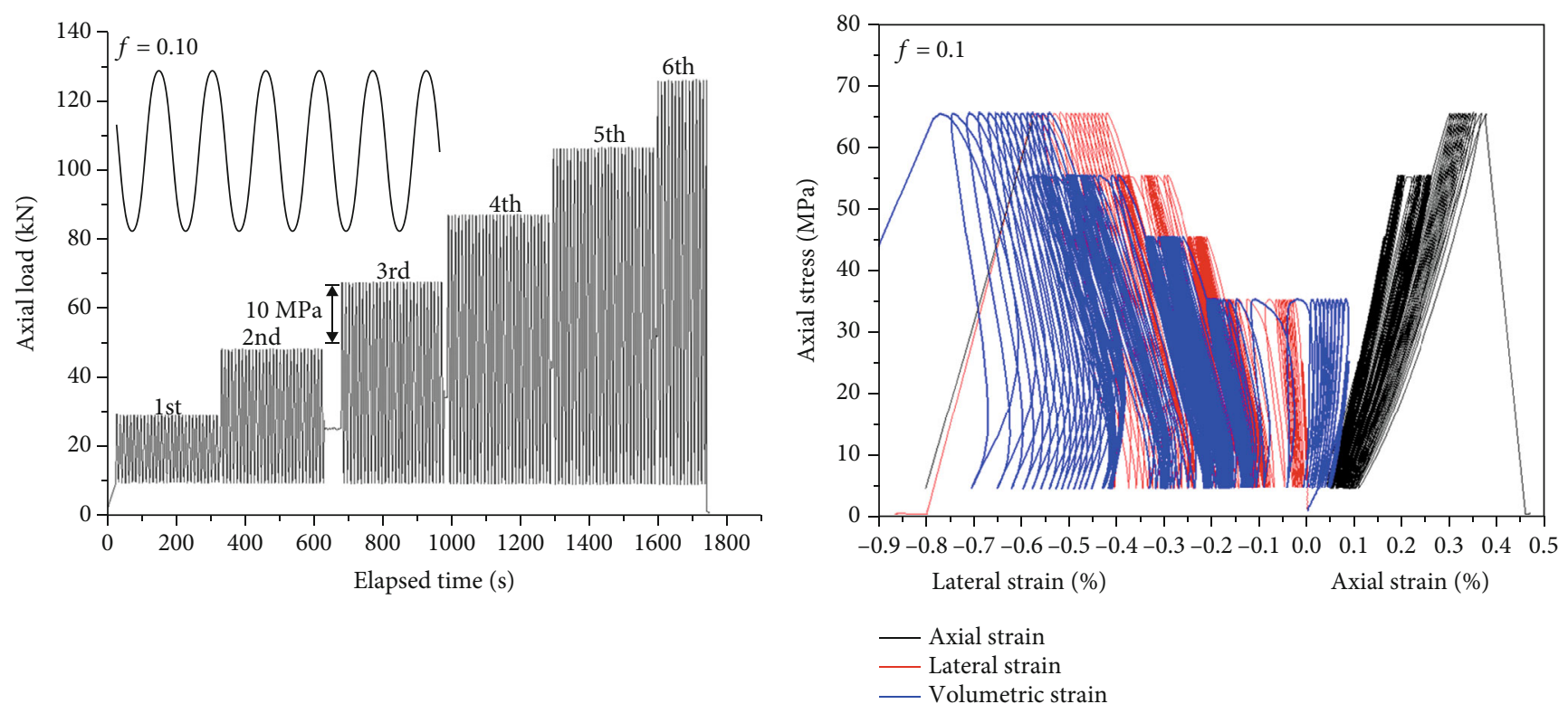

(c)

(d)

Figure 3: Continued. 

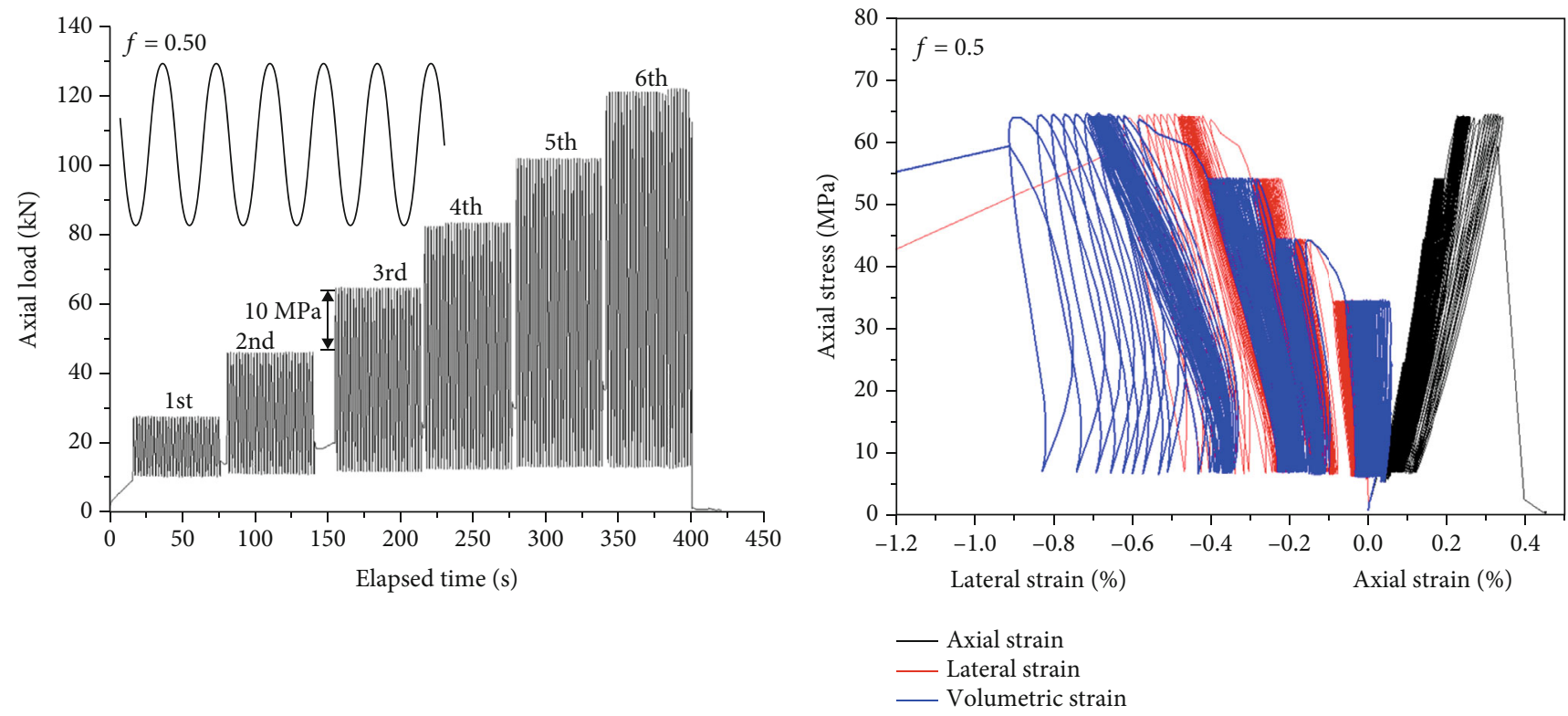

(e)
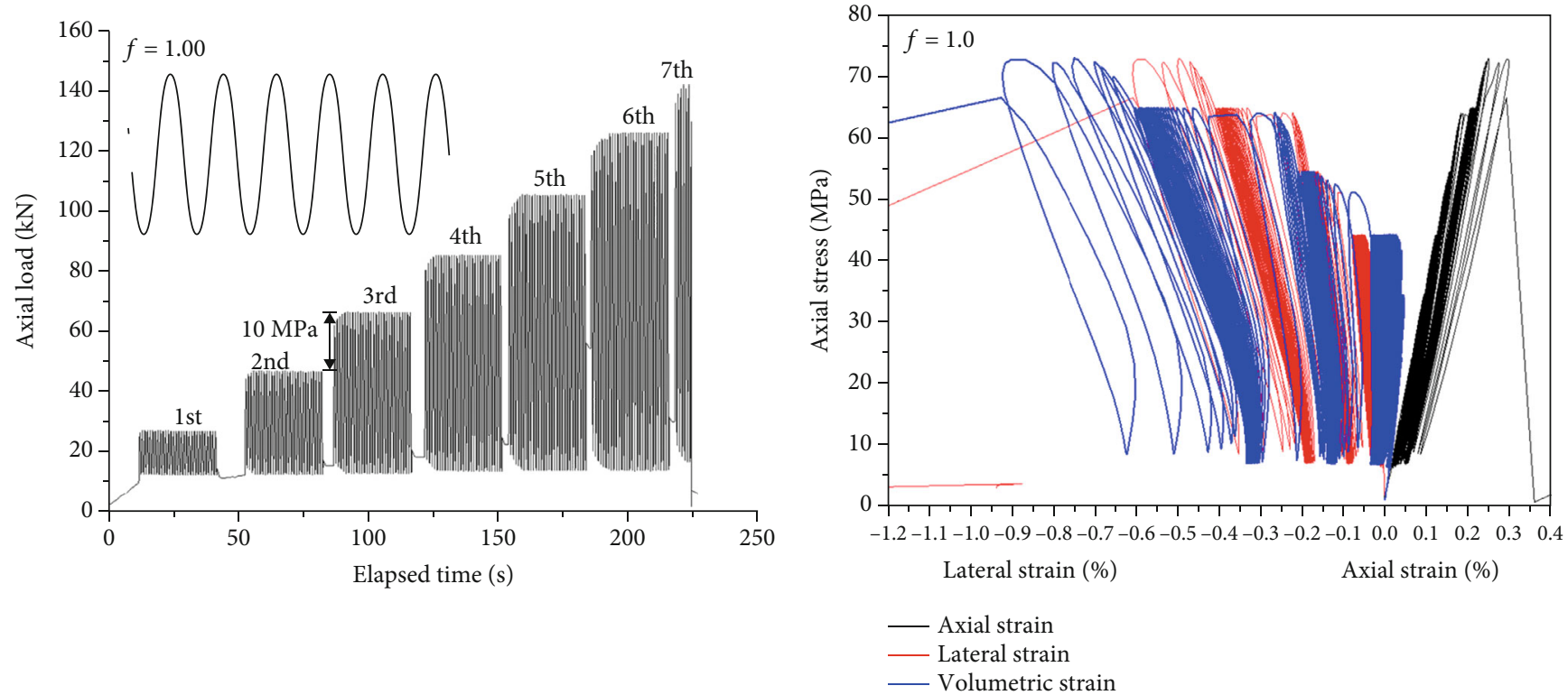

(g)

(h)

FIGURE 3: The loading path and the corresponding complete stress-strain curves for the typical granite samples under dynamic frequency of $0.04,0.1,0.5$, and 1.0, respectively. ( $\mathrm{a}, \mathrm{c}, \mathrm{e}, \mathrm{g}$ ) Plots of the loading path of the cyclic loading. (b, $\mathrm{d}, \mathrm{f}, \mathrm{g}$ ) Plots of the axial, lateral, and volumetric stress-strain curves.

investigation, a dominant joint having an approach angle of $50^{\circ}$ to the rock stratum was observed. According to the method recommended by ISRM, the rock mass obtained from the open pit slope was prepared into a cylinder with a diameter $(D)$ of $50 \mathrm{~mm}$ and a height $(H)$ of $100 \mathrm{~mm}$. Both ends of the sample were polished to ensure that the nonuniformity error is less than $0.05 \mathrm{~mm}$ and the parallelism is less than $0.1 \mathrm{~mm}$.

In order to mimic the rock bridge structure in the rock mass, the cylindrical rock cores with two unparallel flaws were prepared using a water jet system (Figure 1(a)). Highpressure water mixed with garnet abrasive ejected from a
$0.75 \mathrm{~mm}$ diameter nozzle produced a flaw with its aperture of $1 \mathrm{~mm}$ [2]. A detailed method to produce the two flaws can be found at Wang et al. [2]. The flaw geometry in a double-flawed sample was a combination of a horizontal flaw with length $(2 a)$ and an upper inclined flaw $(2 a)$ of $16 \mathrm{~mm}$. The inclined flaw had an approach angle $(\beta)$ of $50^{\circ}$ to the horizontal flaw. The rock bridge length $(2 b)$ (i.e., rock bridge length) as defined in Figure 1(b) was set to be $20 \mathrm{~mm}$ in order to allow the crack coalescence in the locking section.

2.2. Testing Program. The testing device used in this work includes a GCTS RTR 2000 rock mechanics testing machine 


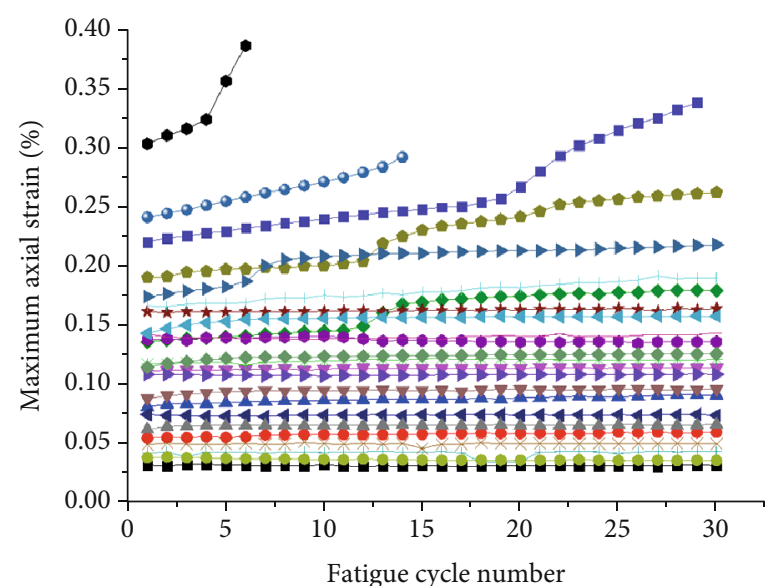

$$
\begin{aligned}
& f=0.04 \quad f=0.1 \quad f=0.5 \quad f=1.0 \\
& - \text { Stage } 1 \multimap \text { Stage } 1 \multimap \text { Stage } 1 \multimap \text { Stage } 1 \\
& \rightarrow \text { Stage } 2 \rightarrow \text { Stage } 2 \rightarrow \text { Stage } 2 \multimap \text { Stage } 2 \\
& - \text { Stage } 3 \rightarrow \text { Stage } 3 \rightarrow \text { Stage } 3 \rightarrow \text { Stage } 3 \\
& \rightarrow \text { Stage } 4 \rightarrow \text { Stage } 4 \longrightarrow \text { Stage } 4 \multimap \text { Stage } 4 \\
& \leftrightarrow \text { Stage } 5 \multimap \text { Stage } 5 \multimap \text { Stage } 5 \multimap \text { Stage } 5 \\
& \rightarrow \text { Stage } 6 \rightarrow \text { Stage } 6 \rightarrow \text { Stage } 6
\end{aligned}
$$

(a)

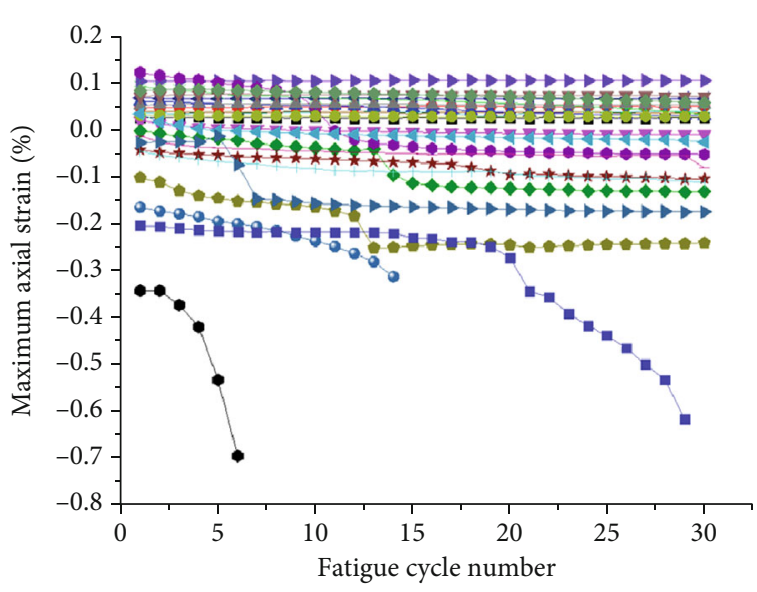

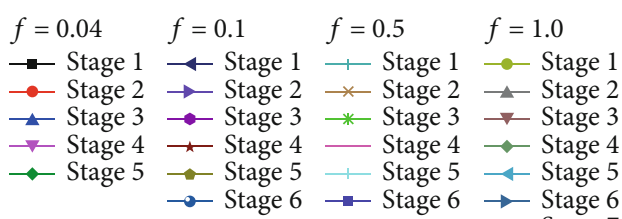

(c)

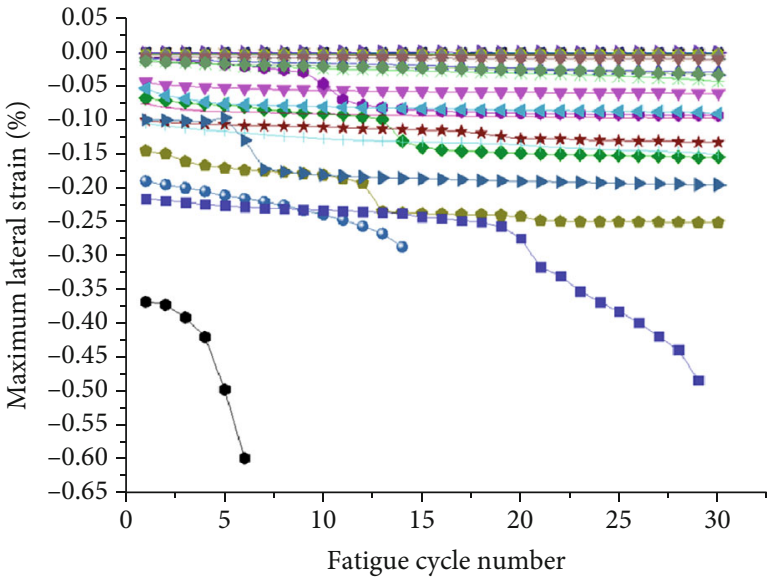

$$
\begin{aligned}
& f=0.04 \quad f=0.1 \quad f=0.5 \quad f=1.0 \\
& \rightarrow \text { Stage } 1 \multimap \text { Stage } 1 \multimap \text { Stage } 1 \multimap \text { Stage } 1 \\
& \rightarrow \text { Stage } 2 \rightarrow \text { Stage } 2 \rightarrow \text { Stage } 2 \multimap \text { Stage } 2 \\
& \rightarrow \text { Stage } 3 \rightarrow \text { Stage } 3 \rightarrow \text { Stage } 3 \rightarrow \text { Stage } 3 \\
& \rightarrow \text { Stage } 4 \multimap \text { Stage } 4 \multimap \text { Stage } 4 \multimap \text { Stage } 4 \\
& \multimap \text { Stage } 5 \multimap \text { Stage } 5 \multimap \text { Stage } 5 \multimap \text { Stage } 5 \\
& \rightarrow \text { Stage } 6 \rightarrow \text { Stage } 6 \rightarrow \text { Stage } 6
\end{aligned}
$$

(b)

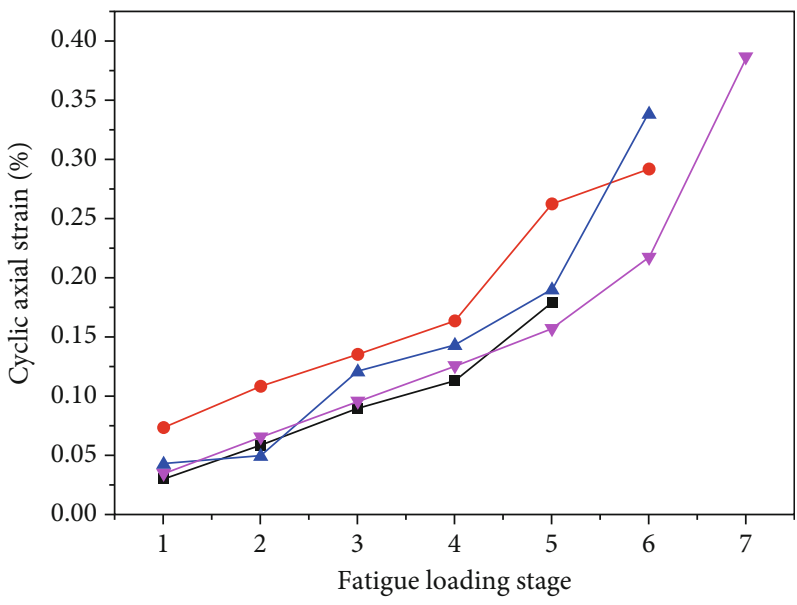

$$
\begin{aligned}
\rightarrow f & =0.04 & \rightarrow f & =0.5 \\
\longrightarrow f & =0.1 & \rightarrow f & =1.0
\end{aligned}
$$




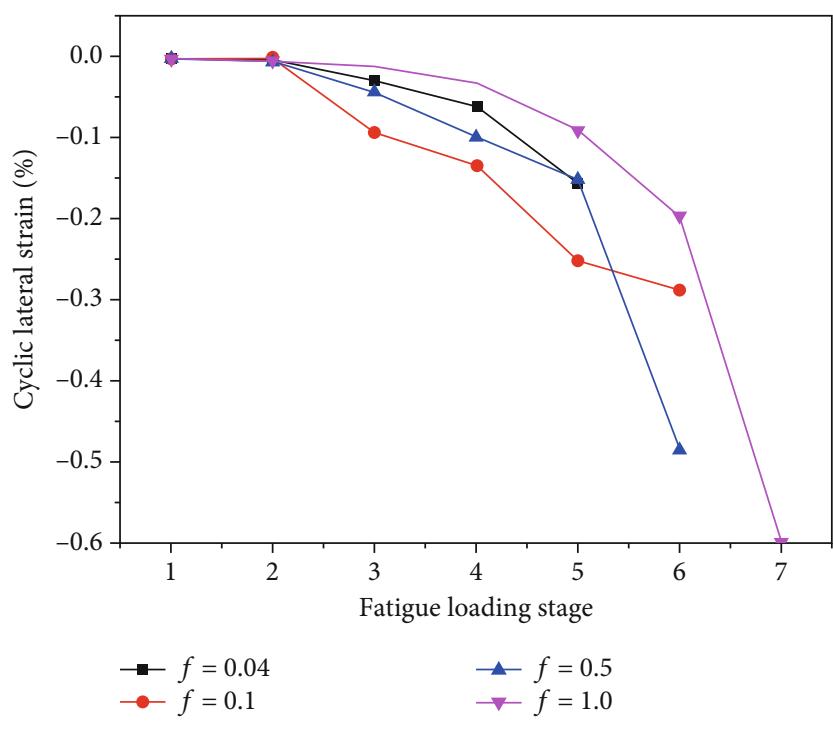

(e)

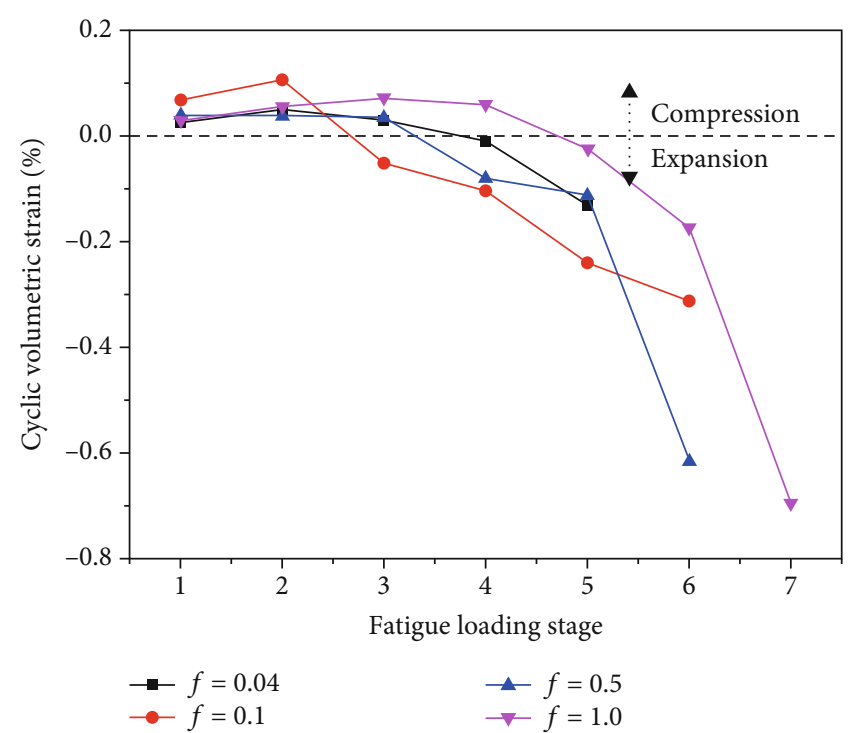

(f)

FIgURE 4: The relationship between the maximum strain and dynamic cyclic number. (a-c) Plots of the maximum axial strain (a), maximum lateral strain (b), and maximum volumetric strain (c) against cyclic number at a fatigue loading stage. (e, f) Plots of the cyclic axial, lateral, and volumetric strain against fatigue loading stage.

and a PAC acoustic emission (AE) apparatus made by the Physical Acoustic Corporation Ltd., USA, as shown in Figure 2. The dynamic loading frequency of the rock mechanics machine is $0 \sim 10 \mathrm{~Hz}$. The PAC AE system is used to monitor the whole cracking process. Detailed description of the $\mathrm{AE}$ system can be found in the literature of Wang et al. [2]. The AE waveform, AE counts, energy, frequency, amplitude, rise time, etc., are recorded during sample deformation.

For the granite samples that have two preexisting flaws, two testing types were conducted, i.e., (1) conventional uniaxial static compression test and (2) uniaxial increasingamplitude stress-cycling tests. For the static loading test, a constant strain rate of $0.06 \mathrm{~mm} / \mathrm{min}$ (i.e., $1.0 \times 10^{-5} \mathrm{~s}^{-1}$ ) was applied to granite samples. For the dynamic increasing amplitude stress-cycling experiments, from the monitoring data of blast variation and cyclic tramcar loading, the dynamic frequency is varied and not constant; four kinds of dynamic frequencies were applied to the flawed granite samples, i.e., $0.04,0.1,0.5$, and $1.0 \mathrm{~Hz}$. First of all, the sample was loaded to a stress of $5 \mathrm{MPa}$ at a constant strain rate $0.06 \mathrm{~mm} / \mathrm{min}$ (i.e., $1.0 \times 10^{-5} \mathrm{~s}^{-1}$ ); then, cyclic dynamic loading was loaded with a certain loading frequency (i.e., 0.04, $0.1,0.5$, and $1.0 \mathrm{~Hz}$ ). During the fatigue loading process, for the first cyclic dynamic loading stage, a stress-controlled sinusoidal cyclic load having a stress amplitude of $10 \mathrm{MPa}$ was applied. In each subsequent cyclic loading stage, the stress amplitude was increased by $10 \mathrm{MPa}$ and also controlled by a sinusoidal cyclic loading type. Stress cycling was continued in this way until the sample eventually failed. For each dynamic cyclic loading stage, 30 stress cycles were applied to the granite sample. Two groups of samples were tested; in group I, they experienced monotonous uniaxial loading; in group II, they are subjected to increasing amplitude stress-cycling conditions, as listed in Table 1.

\section{Result Analysis}

3.1. Typical Cyclic Stress-Strain Responses. For the samples under different dynamic loading frequencies, the loading paths and the associated completed stress-strain curves are plotted in Figure 3. Figures 3(a), 3(c), 3(e), and 3(g) show that different fatigue loading stages were performed for the samples; they are 5, 6, 6, and 7 stages, respectively. It also shows that the number of loading cycle is different for the four samples due to the difference of loading frequency. The total loading cycle number for the four tested samples is 150 , 162,177 , and 190, respectively. The loading cycle number increases with the increasing dynamic loading frequency, which indicates that the frequency impacts the strength, deformation, and the associated fracturing characteristics. The axial stress-strain curves show that obvious brittle failure occurs at the peak stress point; the strain is less than $0.5 \%$ for the samples with different dynamic loading frequencies. Plastic deformation occurs inside rock samples with the increase of experimental time; it can be shown that the loading curve was not overlapped with the unloading curve; therefore, hysteresis loop forms. In order to well describe the impact of axial and lateral deformation on the sample volumetric changes, volumetric strain $\left(\varepsilon_{\mathrm{v}}\right)$ is calculated according to the axial strain $\left(\varepsilon_{1}\right)$ and lateral strain $\left(\varepsilon_{3}\right)$ as $\varepsilon_{v}=\varepsilon_{1}+2 \varepsilon_{3}$. The volumetric strain reflects the combination effect of axial and lateral deformation on deformation. From the volumetric strain curve (line with blue color in Figure 3), deformation characteristics at different loading stages can be expressed obviously. The morphology of hysteresis loop changes at 


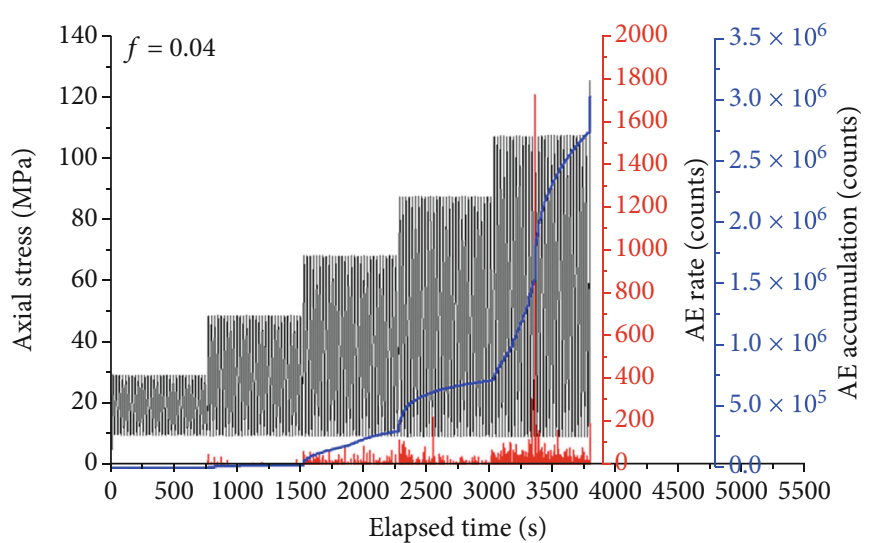

- Stress

AE rate - Accumulative counts

(a)

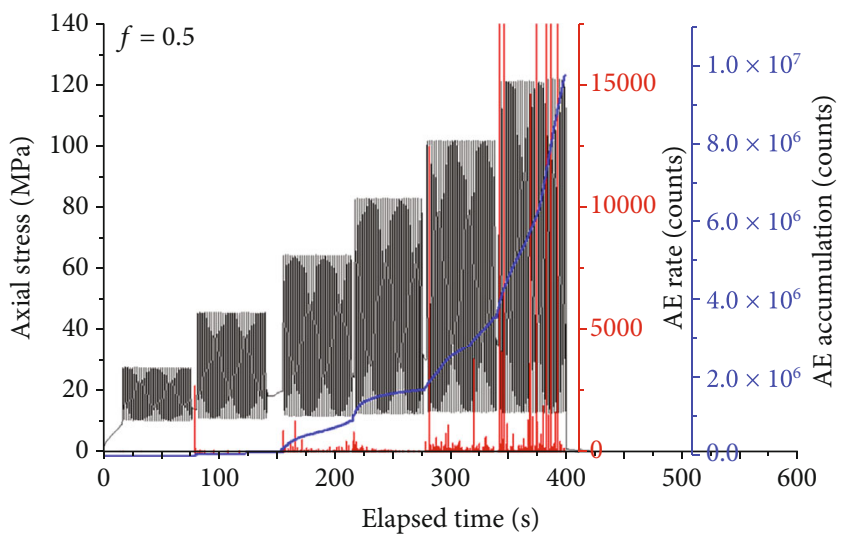

- Stress

AE rate Accumulative counts

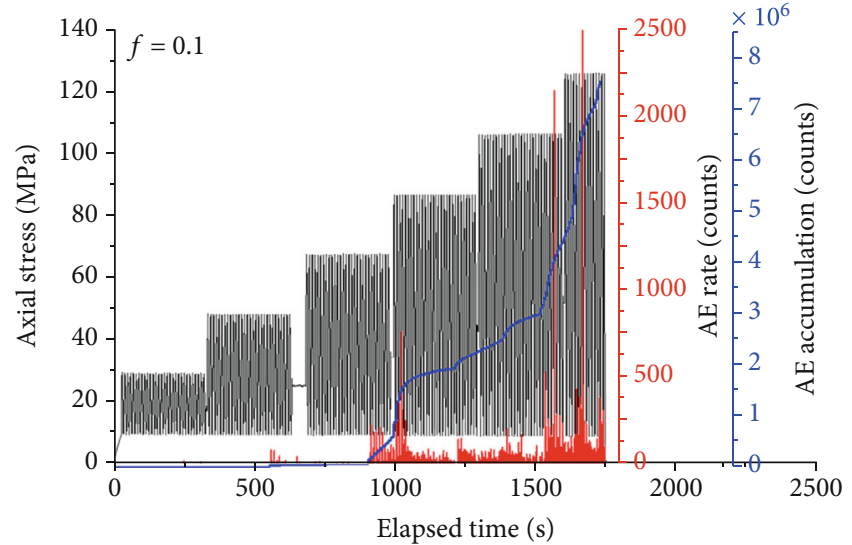

_ Stress

AE rate Accumulative counts

(b)

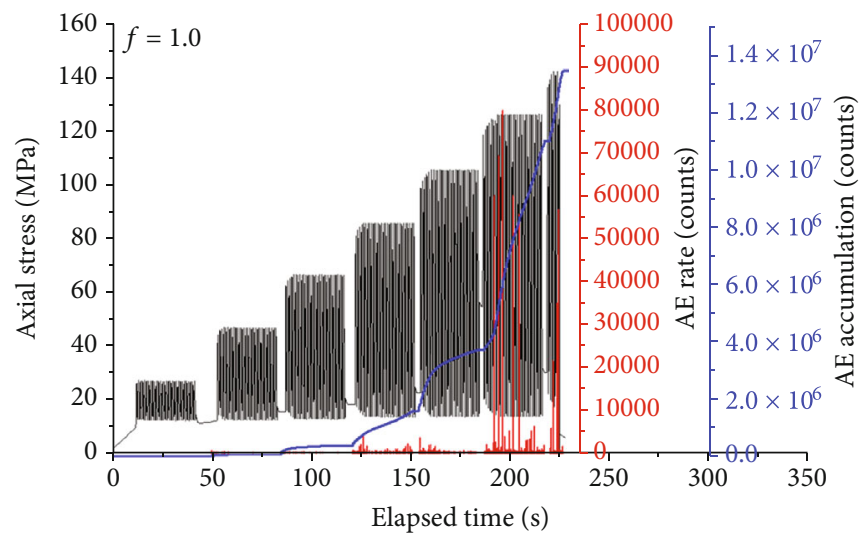

- Stress

AE rate

_ Accumulative counts

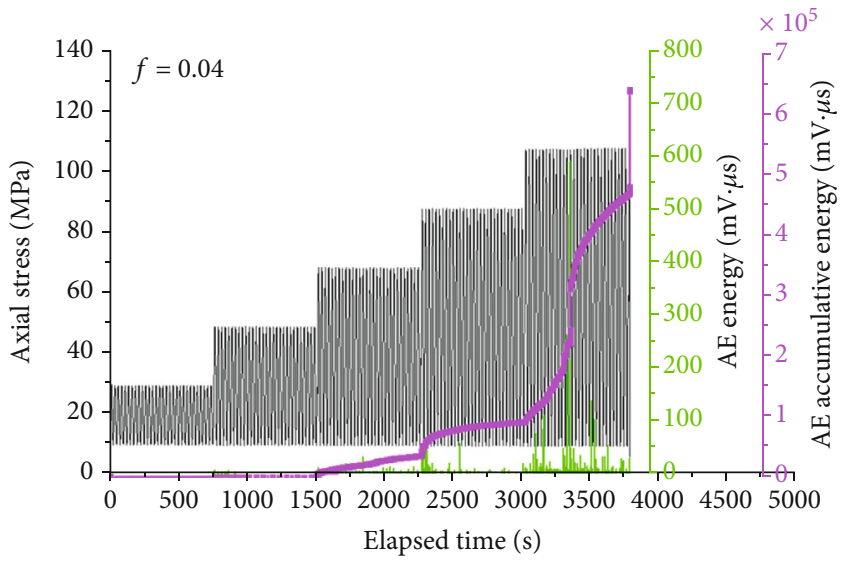

- Stress

Energy

- Accumulative energy

(e)

Figure 5: Continued. 

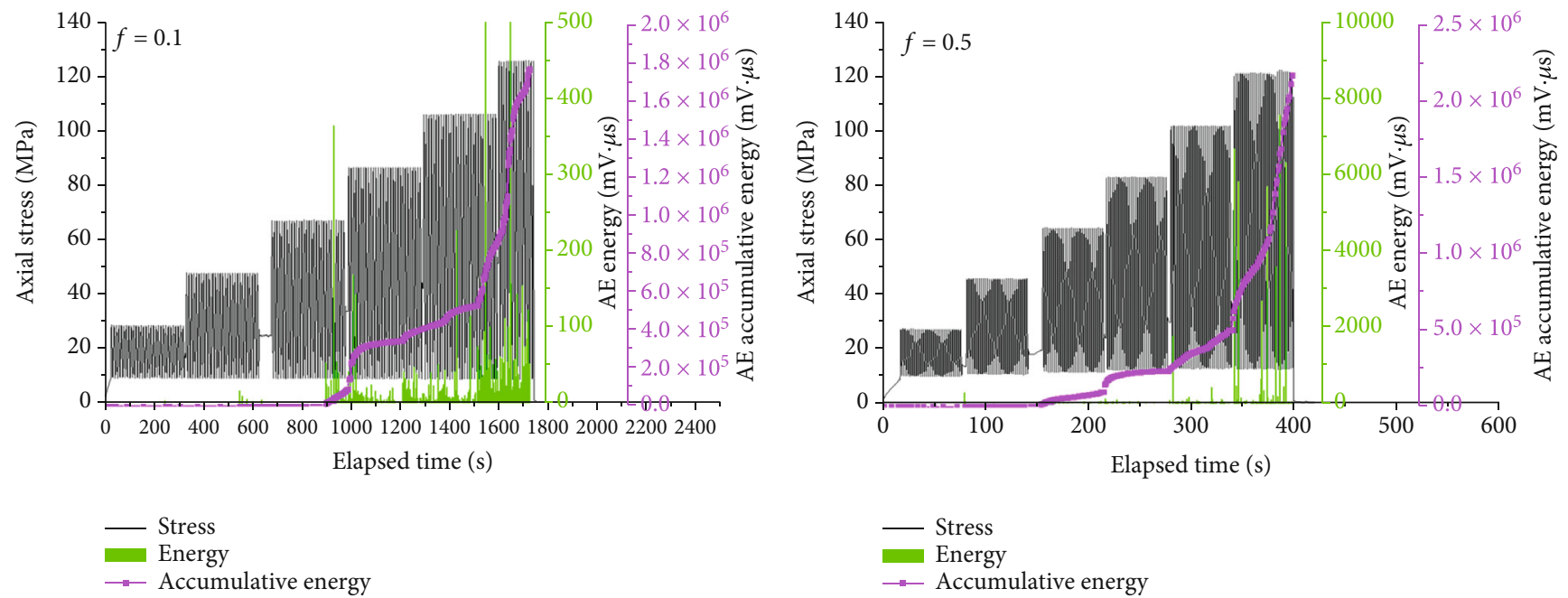

(f)

(g)

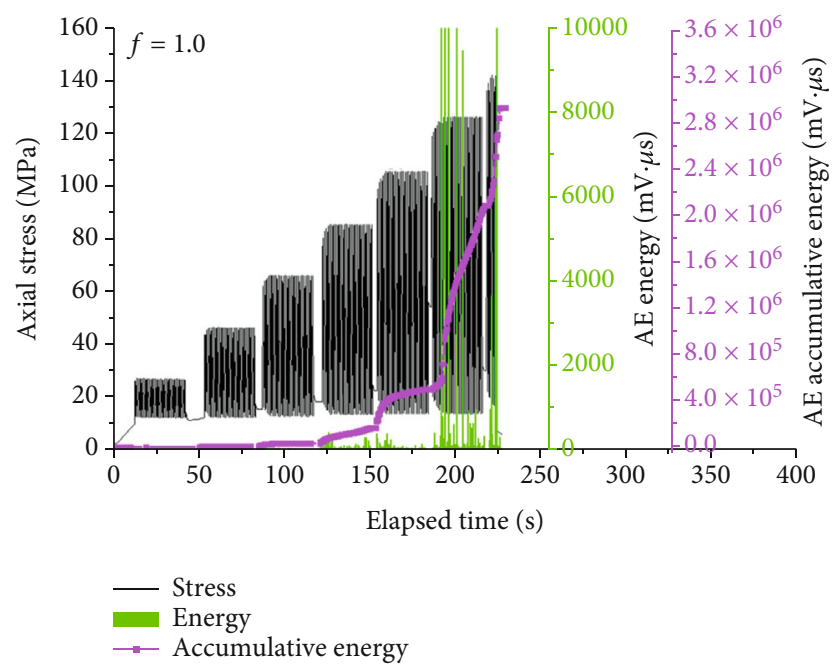

(h)

Figure 5: The relationship between the axial stress, AE rate, AE count accumulation, AE energy, AE accumulative energy, and the experimental time for preflawed granite under dynamic frequency of 0.04, 0.1, 0.5, and 1.0, respectively. (a-d) Description of AE counts during rock deformation. (e-h) Description of AE energy during rock deformation.

different loading time. Before sample failure, hysteresis loop displays as a sparse pattern, and then, it changes to a dense pattern at each fatigue loading stage. The sparse morphology of the hysteresis loop is due to the relatively large plastic deformation resulting from the increase of stress amplitude. After an increased loading stress, the already formed cracks were closed and the morphology of the hysteresis loop changes to dense. At the last fatigue loading stage, changes of the hysteresis loop are different from those before the last loading stage. The hysteresis loop becomes sparser and sparser until failure of the sample. The morphology of the hysteresis loop at each fatigue loading stage reflects the internal mesostructural changes of rock, which is closely related to the propagation behavior of cracks.

3.2. Sample Deformation Characteristics. The rock deformation characteristics during the whole experimental time are shown in Figure 4. From Figures 4(a)-4(c), it can be seen that the axial, lateral, and volumetric strain is small at the low loading cycle number; after a certain loading cycle number, the growth rate of axial, lateral, and volumetric strain becomes faster until failure. Figures 4(d)-4(f) show the relationship between the deformation and fatigue loading stage. They also show that the deformation growth rate of the strain becomes larger with the increase of the loading stage. At the final fatigue loading stage, the axial, lateral, and volumetric strain all increases sharply with increasing dynamic loading frequency. This result indicates that the failure degree and the failure morphology are different for samples under different loading frequencies. Actually, the loading frequency is directly related to the loading rate; the result implies that the loading rate may impact the final failure morphology of rock samples.

3.3. AE Count/Energy Characteristics during Sample Deformation. The stress-strain curves reflect the macroscopic 


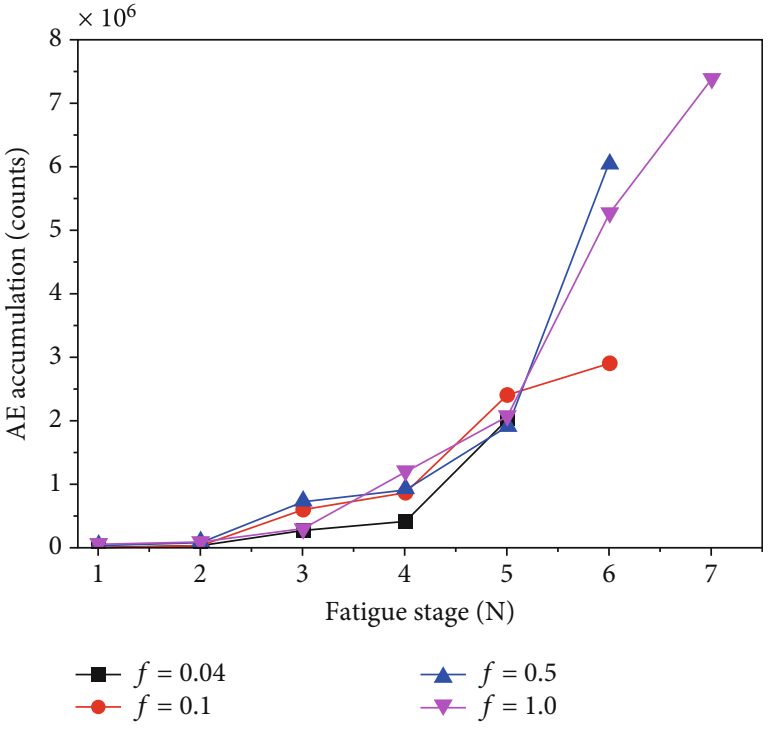

(a)

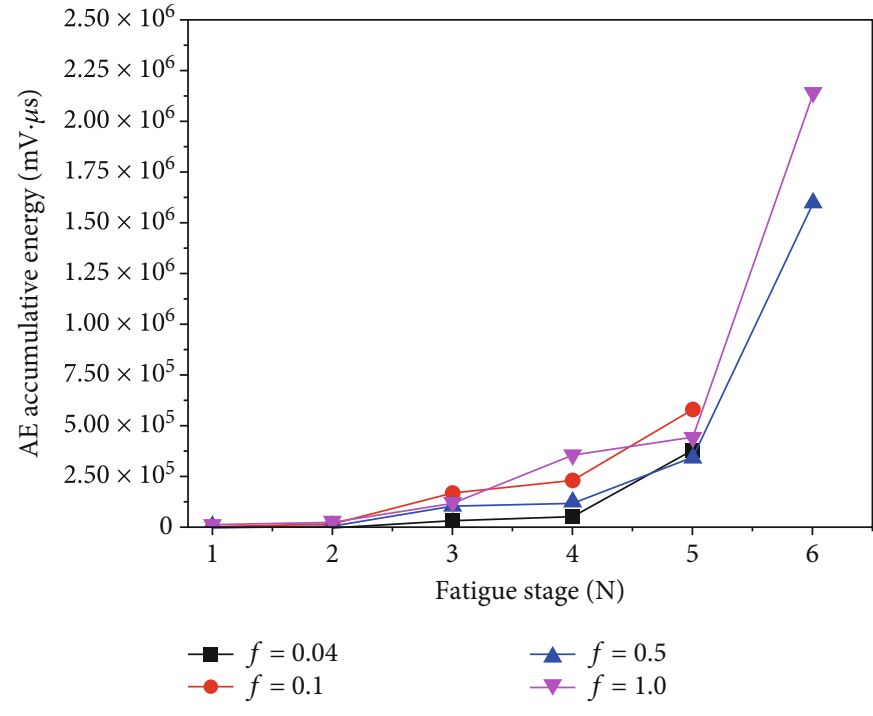

(b)

FIGURE 6: Depict the AE accumulative counts (a) and AE accumulative energy (b) with the fatigue loading stage.

fracturing evolution characteristics of the granite samples. The AE activities recorded during sample deformation provide us insight about the mesoscopic fracturing process of rock [24-26]. The AE output from the four experiments as plotted against stress and experimental time is shown in Figure 5. The AE count or AE energy changes reflect the fracturing evolution characteristics. From the changes of $\mathrm{AE}$ count in Figures 5(a)-5(d), obvious AE count skips at the onset of one fatigue loading stage. This indicates the further propagation of cracks inside the rock sample with the increase of axial stress. Within one fatigue loading stage, $\mathrm{AE}$ activities are relatively less at the first few loading cycles; however, $\mathrm{AE}$ activities grow faster at the loading stage near to sample failure. The generation of AE counts in Figures 5(a)5(d) indicates the damage of mesostructures inside the rock sample, and the damage gradually increases with loading cycles. The pattern of the accumulative AE count curve presents a step shape, indicating the sudden increment of the $\mathrm{AE}$ event at each fatigue loading stage. From the AE accumulation curve, it can be seen that the $\mathrm{AE}$ event increasing rate becomes larger and larger, indicating the accumulative damage in the rock sample, crack initiation, propagation, and coalescence behaviors occuring during the whole loading process. The AE count curve also shows that the generated $\mathrm{AE}$ activities are affected by the loading frequency. For the rock sample with a loading frequency of 0.04 and $0.1 \mathrm{~Hz}$, it shows that the $\mathrm{AE}$ count response is relatively weak, and the generated $\mathrm{AE}$ event is less. However, for the sample with a loading frequency of 0.5 and $1.0 \mathrm{~Hz}$, it can be seen that plenty of $\mathrm{AE}$ events generate, especially at the loading stage close to sample failure.

For the changes of $\mathrm{AE}$ energy against stress and experimental time are shown in Figures 5(e)-5(h), similar conclusions can also be drawn. The sudden increase of AE energy indicates the increment of accumulative damage. The AE energy curve skips at the onset of one fatigue loading stage.
At each loading stage, the released AE energy continuously increases and the increasing trend become more and more obvious. The $\mathrm{AE}$ accumulative energy curves indicate that the loading frequency influences the released energy during sample deformation.

To further reveal the impact of loading frequency on the $\mathrm{AE}$ activities, Figure 6 plots the relationship between the accumulative AE count/energy and experimental time. It can be seen that the increment of the $\mathrm{AE}$ activities increases with the increases of the loading stage. In addition, loading frequency has an obvious influence on the generation of $\mathrm{AE}$ events, and samples subjected to high loading frequency have larger $\mathrm{AE}$ activities than those subjected to relatively low loading frequency.

3.4. Analysis of AE Spectrum Frequency. The above sections are focused on observing the AE activity by counting the event rate (or the cumulative numbers) and observing $\mathrm{AE}$ energy (or the cumulative energy); these analyses are based on the $\mathrm{AE}$ event parameters. The AE signals consisted of a variety of frequency components, and the waveform frequency and amplitude are different for different $\mathrm{AE}$ sources [27-31]. The waveform characterization can reflect the crack type, number, and scale; therefore, the spectrum (frequency) information can well reflect the fracture evolution of rock. Some scholars have also found that the AE main frequency characteristics can better reflect the microfracturing of rock, and it is much more sensitive to the AE counts $[29,30]$. Investigation of the frequency characteristics of emission events, on which our attention will be focused in this section, could be significant and promising as a means of characterizing the effects of dynamic loading frequency on the fracture of rock bridge. The extracted discrete time domain signal can be transferred to the continuous frequency domain signal using Fast Fourier Transform (FFT) Algorithm transformation [30]. Using FFT analysis, the 


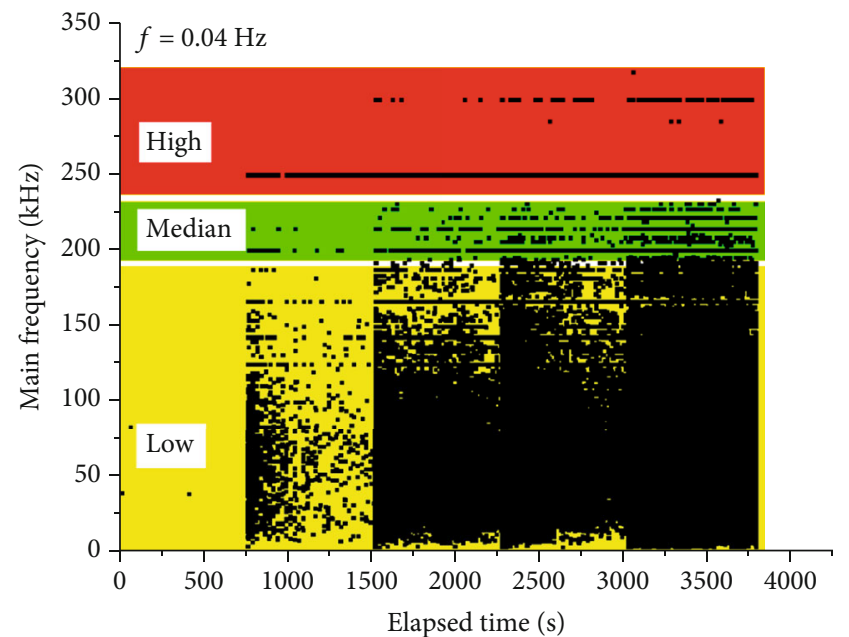

(a)

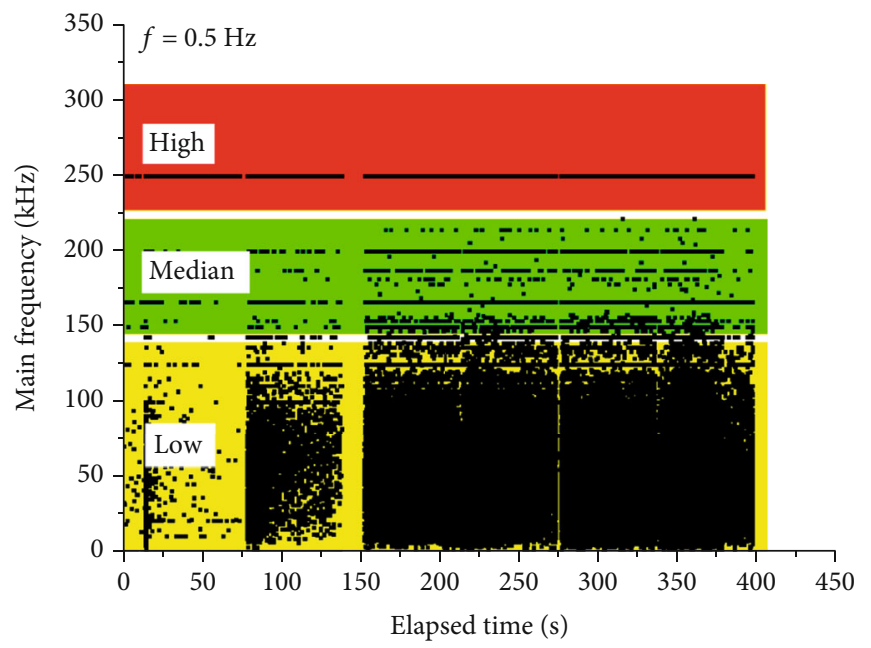

(c)

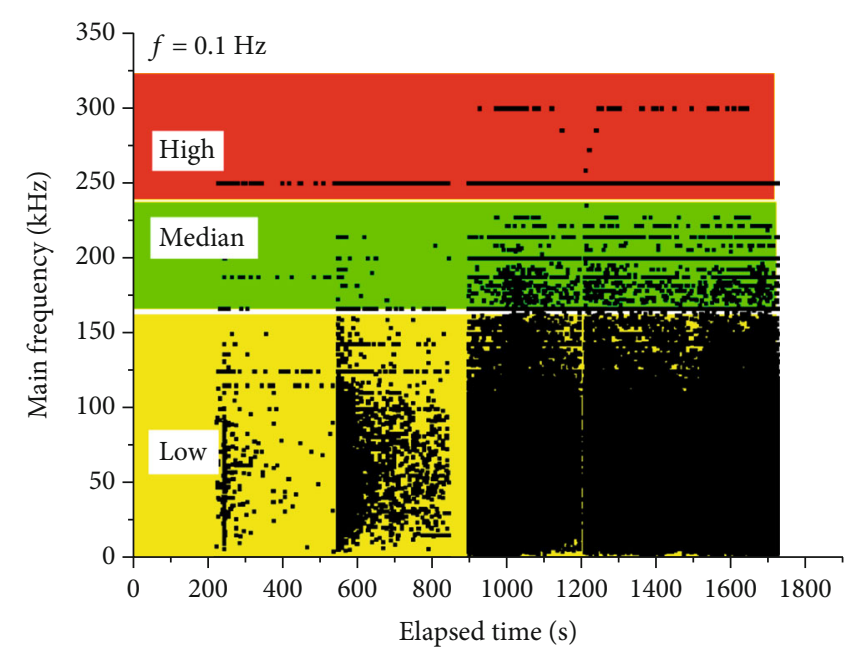

(b)

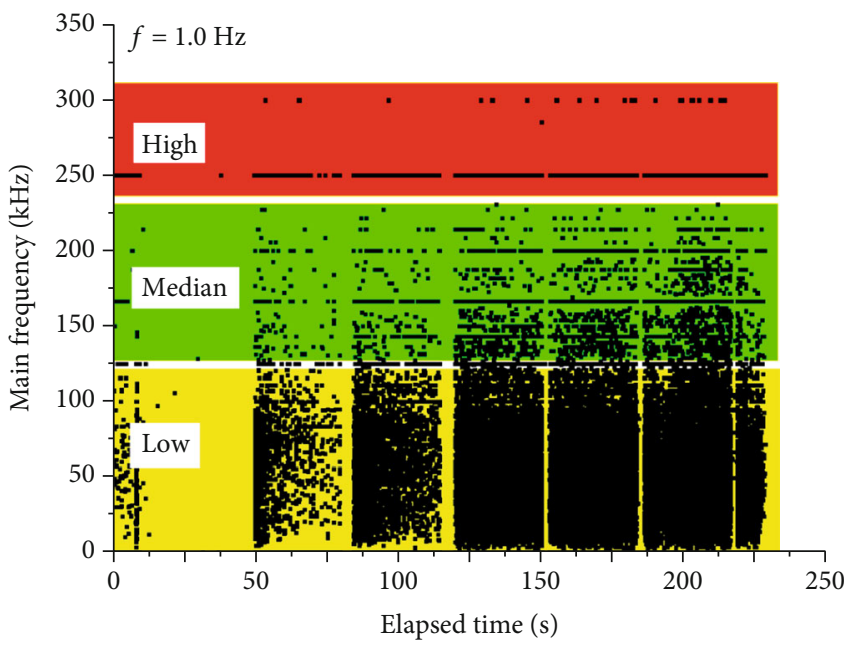

(d)

Figure 7: The main frequency characteristics of the tested samples during fracturing. (a-d) The dynamic loading frequency is $0.04,0.1,0.5$, and 1.0 , respectively.

TABLE 2: Main frequency distribution range for the tested samples with different dynamic loading frequencies.

\begin{tabular}{lccc}
\hline Loading frequency $(\mathrm{Hz})$ & Low & Medium & High \\
\hline 0.04 & {$[0,195]$} & {$[195,230]$} & {$[230,320]$} \\
0.1 & {$[0,160]$} & {$[160,240]$} & {$[240,320]$} \\
0.5 & {$[0,140]$} & {$[140,225]$} & {$[225,320]$} \\
1.0 & {$[0,120]$} & {$[120,235]$} & {$[235,320]$} \\
\hline
\end{tabular}

frequency distribution is shown in Figure 7. Three frequency band ranges of low, medium, and high can be divided, as listed in Table 2.

From the scatter of the main frequency points, it can be found that the frequency distribution is dense at the onset of each fatigue loading stage. This result is consistent with the result of AE count/energy analysis. The dense characteristic implies the occurrence of plenty of fracturing events. In addition, the number of low-frequency signals is more at the beginning of each fatigue loading stage. The medium- and high-frequency signals gradually generate at the last few of the loading stages, and its distribution is impacted by the loading frequency. The scale of the high-frequency signals increases with increasing loading frequency. This result indicates that the released energy is high when the sample is close to the failure state.

As AE amplitude has certain correction with released energy and also the spectrum frequency characteristics, the division of the AE main amplitude can make us better investigate the impact of dynamic loading frequency on the sample fracturing mechanism. The examination of the $\mathrm{AE}$ amplitude distribution makes it possible to monitor the instability of brittle rock [32-34]; it has been proved that the fracture energy have correlations with the AE amplitude. In the spectrum figures, the maximum amplitude value is defined as the main frequency amplitude value, which is used to characterize the intensity of the acoustic emission event, and the frequency corresponding to the main amplitude is defined as the main frequency. The effect of the dynamic loading frequency on the AE main amplitude (which 


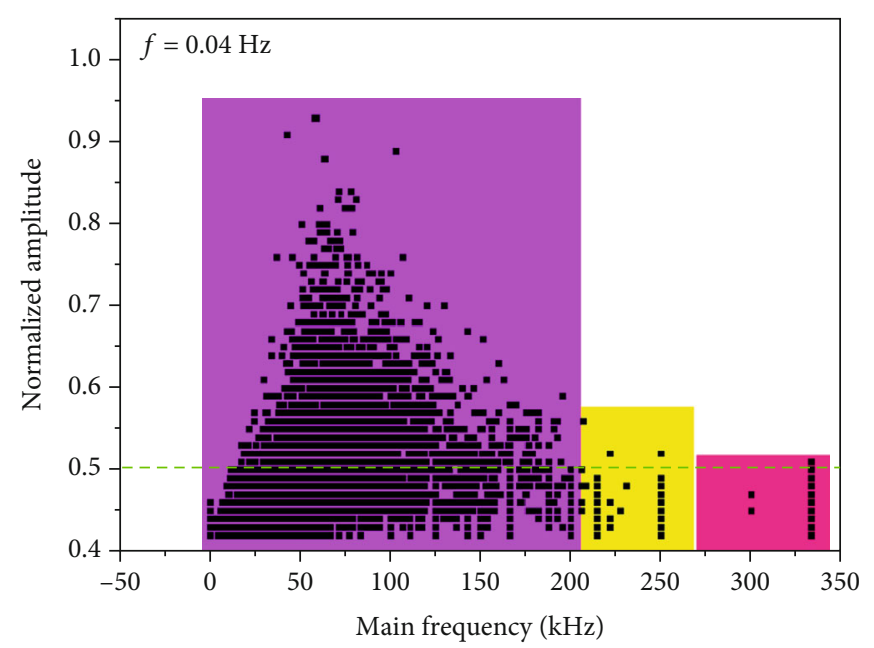

(a)

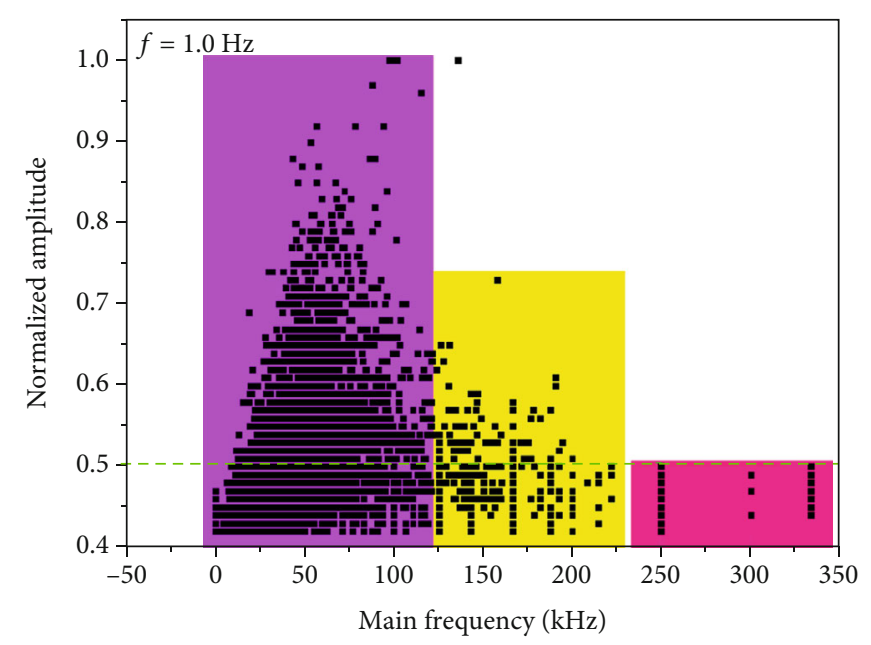

(b)

FIGURE 8: Plots of the normalized amplitude against the main frequency of AE signals. (a) The dynamic loading frequency is $0.04 \mathrm{~Hz}$. (b) The dynamic loading frequency is $1.0 \mathrm{~Hz}$.

corresponds to the main frequency in an AE event) distribution is shown in Figure 8. It can be seen that the normalized amplitude can be divided into two parts: one part is more than 0.5 , and the other part is less than 0.5 . The result indicates that the distribution of $\mathrm{AE}$ main frequency in an $\mathrm{AE}$ event should introduce the influence of the AE main amplitude to clarify the failure mechanism.

Considering the AE main frequency and main amplitude distribution characteristics in Figures 7 and 8, the AE signals are divided into six types: the low-frequency-low-amplitude (LF-LA) signal, the medium-frequency-low-amplitude (MF-LA) signal, the high-frequency-low-amplitude (HFLA) signal, the low-frequency-high-amplitude (LF-HA) signal, the high-frequency-medium-amplitude (HF-MA) signal, and the high-frequency-high-amplitude (HF-HA) signal. The AE studies on the fracture process of the rock material have shown the stress waves in the form of an acoustic emission (AE) signals which can give insights into the process of energy dissipation and emission in response to the initiation and propagation of crack. Figure 9 plots the six types of spectrum frequency and the axial stress against axial strain. At the initial loading stage, the type of the AE signals is mainly the low-frequency-low amplitude and the high-frequency-low-amplitude signals. At the initial loading stage, the type of the AE signals is mainly the lowfrequency-low-amplitude and the high-frequency-lowamplitude signals. At the crack initiation and unstable propagation stage, the six kinds of signals coexist, and the AE signals move into the median frequency band; this indicates the occurrence of macroscopic cracks. In addition, the number of low-frequency-low-amplitude and low-frequency-highamplitude signals gradually increase at the crack unstable propagation stage. Furthermore, the emerging lowfrequency-high-amplitude signal gradually decreases at the crack coalescence stage. From the frequency distribution characteristics in Figure 9, main conclusions can be drawn as follows: (1) low-frequency-low-amplitude signals exist through the whole fracturing process; they correspond to the intergranular cracks at the initial loading stage and the microcracks during fracturing. (2) The medium-frequencylow-amplitude AE signals generate during the whole fracturing process; they correspond to microcracks during sample fracturing. (3) The low-frequency-high-amplitude signals occur at the last few fatigue loading stage; they correspond to the occurrence of large cracks. (4) The mediumfrequency-high-amplitude signals occur at the last few loading stages; they correspond to the instable propagation of cracks and the sliding of the fracturing plane. (5) The high-frequency-high-amplitude signals correspond to the medium-scaled crack and the transgranular fracturing crack.

As there is an inverse relationship between the AE frequency and the crack size, generally, large-scaled cracks correspond to low-frequency signals, and small-scaled crack corresponds to high-frequency signals. The main frequency distribution in Figure 9 shows that the high-frequencyhigh-amplitude signals do not exist for samples subjected to 0.5 and 1.0 loading frequency. This result indicates that the number of small-scaled cracks decreases for the rock sample under relatively high loading frequency. To better reflect the influence of dynamic loading frequency on the proportion of the low, medium, and high AE signals, the statistic result is listed in Table 3.

\section{Discussions}

Extensive investigations about the mechanical behaviors of rock under cyclic and fatigue loading conditions have been performed [5-10]. However, most of the loading condition is the constant stress amplitude; in addition, they are mainly focused on the macroscopic cracking process of intact rock, especially the crack coalescence pattern description on the intact rock after the test. In addition, rock fracturing behavior under cyclic loads is different from the static loading condition [24, 35-38]. Actually, in rock engineering, the amplitude of the disturbance stress is varied and not constant. Accumulative damage resulting from the increase of stress amplitude 


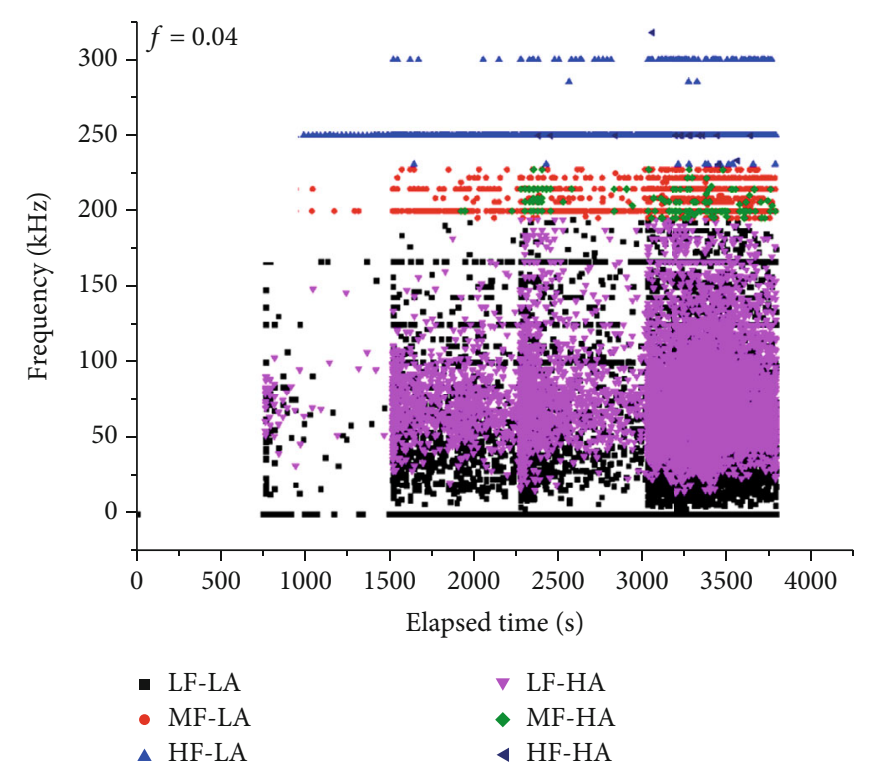

(a)

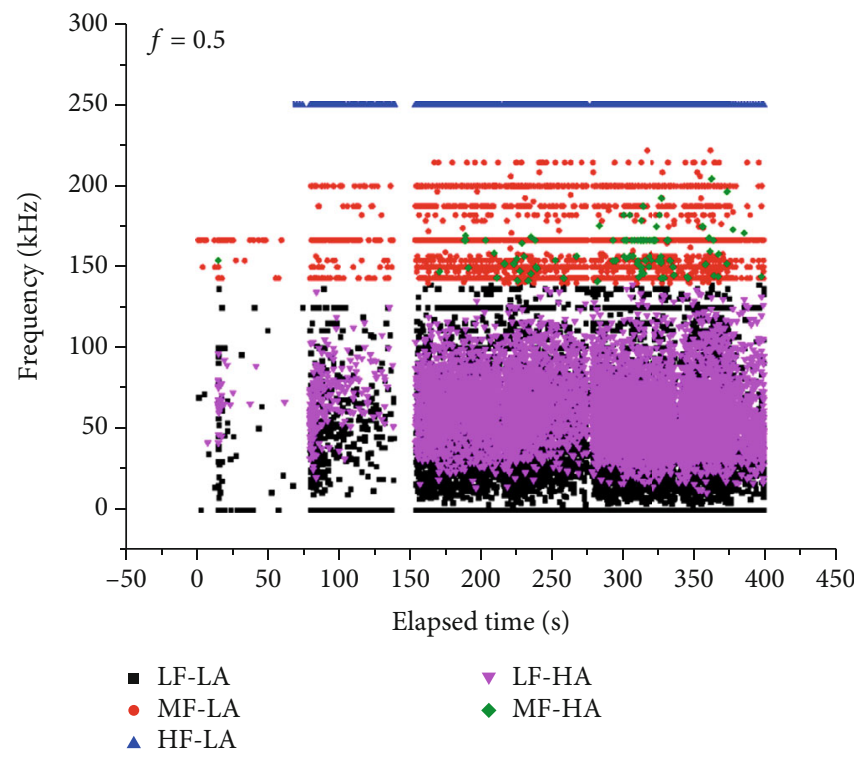

(c)

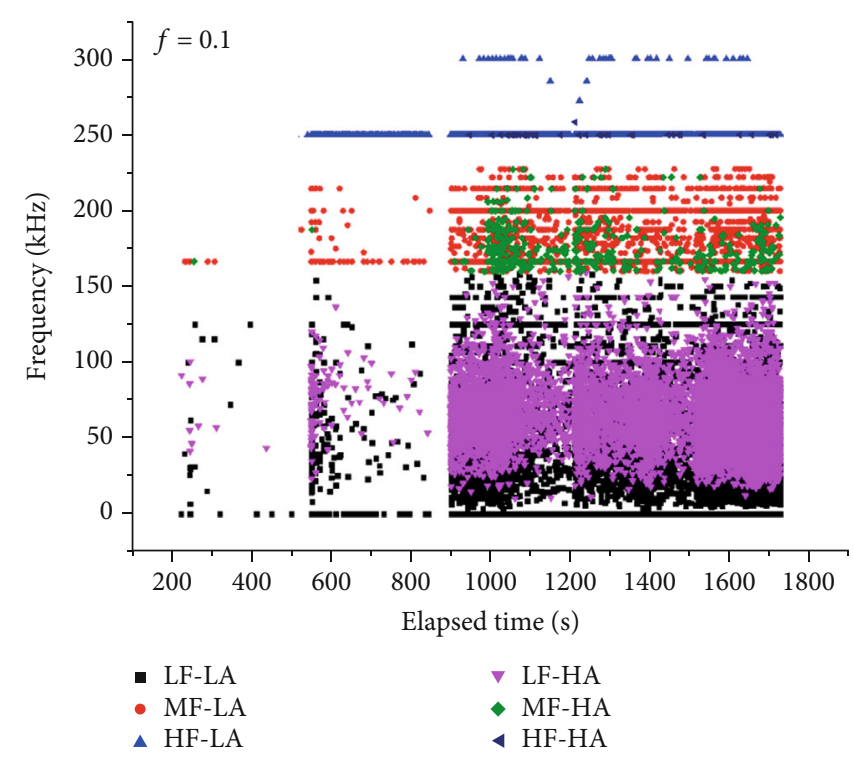

(b)

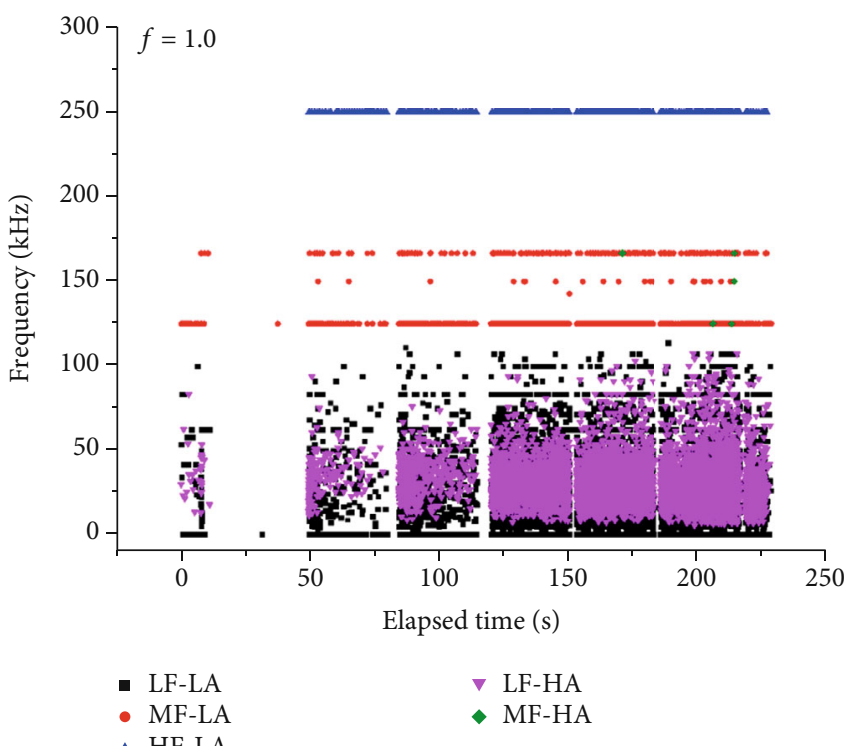

(d)

FIGURE 9: The main frequency characteristics of granite samples during the whole deformation process, during sample failure for granite with inclined flaw of $20^{\circ}, 50^{\circ}$, and $70^{\circ}$, respectively. (a-d) The dynamic loading frequency is $0.04,0.1,0.5$, and 1.0 , respectively.

Table 3: Percentage of the low-, medium-, and high-frequency AE signals during rock deformation.

\begin{tabular}{lccc}
\hline $\begin{array}{l}\text { Loading } \\
\text { frequency } \\
(\mathrm{Hz})\end{array}$ & $\begin{array}{c}\text { Ratio of low } \\
\text { frequency (\%) }\end{array}$ & $\begin{array}{c}\text { Ratio of median } \\
\text { frequency (\%) }\end{array}$ & $\begin{array}{c}\text { Ratio of high } \\
\text { frequency (\%) }\end{array}$ \\
\hline 0.04 & $55 \%$ & $11 \%$ & $34 \%$ \\
0.1 & $62 \%$ & $12 \%$ & $26 \%$ \\
0.5 & $71 \%$ & $11 \%$ & $18 \%$ \\
0.1 & $80 \%$ & $10 \%$ & $10 \%$ \\
\hline
\end{tabular}

on rock occurs in most of rock engineering. By performing fatigue loading experiment on granite samples containing two preexisting flaws, the effect of dynamic loading frequency on the whole fracturing evolution is deeply investigated. The dynamic loading frequency impacts the stressstrain responses, AE responses, and the associated final crack coalescence pattern. The influence of loading frequency on rock mechanical behaviors is actually the influence of the loading rate on the rock. The strength, deformation, and failure morphology of rock is related to loading frequency. Our experimental results also found that the fatigue loading cycle number increases with the increase of loading frequency. The $\mathrm{AE}$ activities increase with increasing loading frequency, and 
the sudden increase of axial stress at the onset of each fatigue loading stage results in relatively large damage accumulation in rock. Due to the differential loading frequencies, the AE counts, and AE energy, the main amplitude distribution and the frequency spectrum characteristics present obvious difference. As is known, AE events are the external reflection of the energy dissipation and release during rock deformation; the velocity of energy release is positively related to the loading rate. For rock subjected to high loading frequency, the associated energy release velocity is relatively high, and the AE counts/energy (or accumulative number) is larger than rock subjected to low loading frequency. As the frequency and amplitude of AE events are much more sensitive, frequency spectrum analysis further reveals the crack propagation and rock bridge fracturing characteristics; cracks generated from the fracture process are classified into six kinds. The experimental results show that the highfrequency-high-amplitude signals do not exist for samples subjected to high loading frequency. Because the highamplitude-high-frequency signals correspond to the formation of small-scaled cracks, this result indicates that the number of small-scaled cracks decreases for the rock sample under relatively high loading frequency. This result implies that the rock structure is easily degraded when subjected to high-stress disturbance frequency.

\section{Conclusions}

In this work, an acoustic emission monitoring technique was used to investigate the effect of dynamic loading frequency on the rock bridge fracturing of preflawed granite samples. From the above analysis, the following conclusions can be drawn:

(1) The deformation, strength, and fatigue lifetime of the preflawed granites are impacted by the applied dynamic loading frequency. Volumetric deformation monitored at the rock bridge area indicates that a sudden rupture occurs at the final cyclic loading stage and the volumetric strain is the maximum for rock subjected to high-frequency loading conditions

(2) AE parameters extracted from the AE waveform are strongly influenced by the applied dynamic frequency. The increasing rate of the $\mathrm{AE}$ count and $\mathrm{AE}$ energy at each loading stage gets faster as the cyclic loading stage increases. The accumulative AE count and energy both increase with the increase of dynamic loading frequency

(3) AE spectral analysis reveals different fracturing mechanisms that are impacted by dynamic frequency. It is suggested that the number of highfrequency-high-amplitude signals decreases for rock subjected to high loading frequency, indicating the occurrence of large-scaled cracks. The main frequency is a good index for early warning of instability prediction for rock with geological discontinuities

\section{Data Availability}

The experimental data used to support the findings of this study are included within the article.

\section{Conflicts of Interest}

The authors declare no conflict of interest.

\section{Acknowledgments}

This study was supported by the China Geological Survey Project (No. DD20190318), the Beijing Science and Technology Project (Z191100001419015), and the Fundamental Research Funds for the Central Universities (FRF-TP-20004A2).

\section{References}

[1] B. Cerfontaine and F. Collin, "Cyclic and fatigue behaviour of rock materials: review, interpretation and research perspectives," Rock Mechanics and Rock Engineering, vol. 51, no. 2, pp. 391-414, 2018.

[2] Y. Wang, J. Q. Han, and C. H. Li, "Acoustic emission and CT investigation on fracture evolution of granite containing two flaws subjected to freeze-thaw and cyclic uniaxial increasingamplitude loading conditions," Construction and Building Materials, vol. 260, article 119769, 2020.

[3] Y. Wang, S. Gao, D. Liu, and C. Li, "Anisotropic fatigue behaviour of interbeded marble subjected to uniaxial cyclic compressive loads," Fatigue \& Fracture of Engineering Materials \& Structures, vol. 43, no. 6, pp. 1170-1183, 2020.

[4] Y. Wang, W. K. Feng, and C. H. Li, "On anisotropic fracture and energy evolution of marble subjected to triaxial fatigue cyclic-confining pressure unloading conditions," International Journal of Fatigue, vol. 134, article 105524, 2020.

[5] Y. Wang, D. Liu, J. Han, C. Li, and H. Liu, "Effect of fatigue loading-confining stress unloading rate on marble mechanical behaviors: an insight into fracture evolution analyses," Journal of Rock Mechanics and Geotechnical Engineering, vol. 121, 2020.

[6] Y. Wang, C. Li, J. Han, and H. Wang, "Mechanical behaviours of granite containing two flaws under uniaxial increasing amplitude fatigue loading conditions: an insight into fracture evolution analyses," Fatigue and Fracture of Engineering Materials and Structures, vol. 43, no. 9, pp. 2055-2070, 2020.

[7] D. K. Liu, Z. L. Gu, R. X. Liang et al., "Impacts of pore-throat system on fractal characterization of tight sandstones," Geofluids, vol. 2020, Article ID 4941501, 17 pages, 2020.

[8] Y. Wang, L. Ma, P. Fan, and Y. Chen, "A fatigue damage model for rock salt considering the effects of loading frequency and amplitude," International Journal of Mining Science and Technology, vol. 26, no. 5, pp. 955-958, 2016.

[9] M. N. Bagde and V. Petroš, "Fatigue properties of intact sandstone samples subjected to dynamic uniaxial cyclical loading," International Journal of Rock Mechanics and Mining Sciences, vol. 42, no. 2, pp. 237-250, 2005.

[10] E. Liu and S. He, "Effects of cyclic dynamic loading on the mechanical properties of intact rock samples under confining pressure conditions," Engineering Geology, vol. 125, pp. 8191, 2012. 
[11] Y. Wang, S. H. Gao, C. H. Li, and J. Q. Han, "Investigation on fracture behaviors and damage evolution modeling of freezethawed marble subjected to increasing-amplitude cyclic loads," Theoretical and Applied Fracture Mechanics, vol. 109, article 102679, 2020.

[12] S. K. Singh, "Fatigue and strain hardening behavior of greywacke from the flagstaff formation, New South Wales," Engineering Geology, vol. 26, no. 2, pp. 171-179, 1989.

[13] S. K. Singh, "Relationship among fatigue strength, mean grain size and compressive strength of a rock," Rock Mechanics and Rock Engineering, vol. 21, no. 4, pp. 271-276, 1988.

[14] X. Ge, "Study of deformation and strength behaviour of the large-sized triaxial rock samples under cyclic loading," Rock and Soil Mechanics, vol. 8, no. 2, pp. 11-18, 1987.

[15] X. R. Ge, Y. Jiang, Y. D. Lu, and J. X. Ren, "Testing study on fatigue deformation law of rock under cyclic loading," Chinese Journal of Rock Mechanics and Engineering, vol. 22, no. 10, pp. 1581-1585, 2003.

[16] K. Fuenkajorn and D. Phueakphum, "Effects of cyclic loading on mechanical properties of Maha Sarakham salt," Engineering Geology, vol. 112, no. 1-4, pp. 43-52, 2010.

[17] Z. Wang, S. Li, L. Qiao, and J. Zhao, "Fatigue behavior of granite subjected to cyclic loading under triaxial compression condition," Rock Mechanics and Rock Engineering, vol. 46, no. 6, pp. 1603-1615, 2013.

[18] L. A. Roberts, S. A. Buchholz, K. D. Mellegard, and U. Düsterloh, "Cyclic loading effects on the creep and dilation of salt rock," Rock Mechanics and Rock Engineering, vol. 48, no. 6, pp. 2581-2590, 2015.

[19] A. Taheri, J. Squires, Z. Meng, and Z. Zhang, "Mechanical properties of brown coal under different loading conditions," International Journal of Geomechanics, vol. 17, no. 11, article 06017020, 2017.

[20] J. Fan, D. Jiang, W. Liu, F. Wu, J. Chen, and J. Daemen, "Discontinuous fatigue of salt rock with low-stress intervals," International Journal of Rock Mechanics and Mining Sciences, vol. 115, pp. 77-86, 2019.

[21] K. Peng, J. Zhou, Q. Zou, and X. Song, "Effect of loading frequency on the deformation behaviours of sandstones subjected to cyclic loads and its underlying mechanism," International Journal of Fatigue, vol. 131, article 105349, 2020.

[22] Y. Wang, S. H. Gao, C. H. Li, and J. Q. Han, "Energy dissipation and damage evolution for dynamic fracture of marble subjected to freeze-thaw and multiple level compressive fatigue loading," International Journal of Fatigue, vol. 142, article 105927, 2021.

[23] K. Lupogo, Characterization of blast damage in rock slopes: an integrated field-numerical modeling approach, [Ph.D thesis], Simon Fraser University, British Columbia, Canada, 2017.

[24] Y. Wang, W. H. Tan, D. Q. Liu, Z. Q. Hou, and C. H. Li, "On anisotropic fracture evolution and energy mechanism during marble failure under uniaxial deformation," Rock Mechanics and Rock Engineering, vol. 52, no. 10, pp. 3567-3583, 2019.

[25] A. Voznesenskii, Y. Kutkin, M. Krasilov, and A. Komissarov, "Predicting fatigue strength of rocks by its interrelation with the acoustic quality factor," International Journal of Fatigue, vol. 77, pp. 194-198, 2015.

[26] A. Voznesenskii, Y. Kutkin, M. Krasilov, and A. Komissarov, "The influence of the stress state type and scale factor on the relationship between the acoustic quality factor and the resid- ual strength of gypsum rocks in fatigue tests," International Journal of Fatigue, vol. 84, pp. 53-58, 2016.

[27] M. Ohnaka and K. Mogi, "Frequency characteristics of acoustic emission in rocks under uniaxial compression and its relation to the fracturing process to failure," Journal of Geophysical Research: Solid Earth, vol. 87, no. B5, pp. 3873-3884, 1982.

[28] M. C. He, J. L. Miao, and J. L. Feng, "Rock burst process of limestone and its acoustic emission characteristics under true-triaxial unloading conditions," International Journal of Rock Mechanics and Mining Sciences, vol. 47, no. 2, pp. 286298, 2010.

[29] Z. Wang, J. Ning, and H. Ren, "Frequency characteristics of the released stress wave by propagating cracks in brittle materials," Theoretical and Applied Fracture Mechanics, vol. 96, pp. 7282, 2018.

[30] Y. Wang, W. Feng, H. Wang, J. Han, and C. Li, "Geomechanical and acoustic properties of intact granite subjected to freeze-thaw cycles during water-ice phase transformation in Beizhan's open pit mine slope, Xinjiang, China," Water, vol. 11, no. 11, p. 2309, 2019.

[31] A. Lavrov, "Kaiser effect observation in brittle rock cyclically loaded with different loading rates," Mechanics of Materials, vol. 33, no. 11, pp. 669-677, 2001.

[32] K. Mogi, "Earthquake prediction program in Japan," in Earthquake Prediction, D. W. Simpson and P. G. Richards, Eds., vol. 4, pp. 635-666, AGU, Washington, D.C., 1981.

[33] K. Mogi, "Magnitude-frequency relation for elastic shocks accompanying fractures of various materials and some related problems in earthquakes (2nd paper)," Bulletin of the Earthquake Research Institute, University of Tokyo, vol. 40, pp. 831-853, 1962.

[34] C. H. Scholz, "Microfracturing and the inelastic deformation of rock in compression," Journal of Geophysical Research, vol. 73, no. 4, pp. 1417-1432, 1968.

[35] J. Wang, Y. Zhang, Z. Qin, S. G. Song, and P. Lin, “Analysis method of water inrush for tunnels with damaged waterresisting rock mass based on finite element method-smooth particle hydrodynamics coupling," Computers and Geotechnics, vol. 126, article 103725, 2020.

[36] J. Xu, A. Haque, W. Gong et al., "Experimental study on the bearing mechanisms of rock-socketed piles in soft rock based on micro X-ray CT analysis," Rock Mechanics and Rock Engineering, vol. 53, no. 8, pp. 3395-3416, 2020.

[37] C. Zhu, M. C. He, M. Karakus, X. B. Cui, and Z. G. Tao, "Investigating toppling failure mechanism of anti-dip layered slope due to excavation by physical modelling," Rock Mechanics and Rock Engineering, vol. 67, 2020.

[38] J. T. Chen, J. H. Zhao, S. C. Zhang, Y. Zhang, F. Yang, and M. Li, "An experimental and analytical research on the evolution of mining cracks in deep floor rock mass," Pure and Applied Geophysics, vol. 33, 2020. 